\title{
Noncommutative scalar field in the nonextremal Reissner-Nordström background: Quasinormal mode spectrum
}

\author{
Marija Dimitrijević Ćirici, ${ }^{1, *}$ Nikola Konjik, ${ }^{1, \dagger}$ and Andjelo Samsarov ${ }^{2, \$}$ \\ ${ }^{1}$ Faculty of Physics, University of Belgrade, Studentski trg 12, 11000 Beograd, Serbia \\ ${ }^{2}$ Rudjer Bošković Institute, Theoretical Physics Division, Bijenička 54, 10002 Zagreb, Croatia
}

(Received 4 September 2019; revised manuscript received 6 April 2020; accepted 21 May 2020; published 15 June 2020)

\begin{abstract}
In our previous work [M. D. Cirić et al., Classical Quantum Gravity 35, 175005 (2018)] we constructed a model of a noncommutative, charged, and massive scalar field based on the angular twist. Then we used this model to analyze the motion of the scalar field in the Reissner-Nordström black hole background. In particular, we determined the quasinormal mode (QNM) spectrum analytically in the near-extremal limit. To broaden our analysis, in this paper we apply a well-defined numerical method, the continued fraction method, and calculate the QNM spectrum for a nonextremal Reissner-Nordström black hole. To check the validity of our analytic calculations, we compare results of the continued fraction method in the near extremal limit with the analytic results obtained in the previous paper. We find that the results are in good agreement. For completeness, we also study the QNM spectrum in the Wentzel-Kramers-Brillouin approximation.
\end{abstract}

DOI: 10.1103/PhysRevD.101.116009

\section{INTRODUCTION}

The study of black hole perturbations has a long history dating back to the work of Regge and Wheeler [1] and Vishveshwara [2]. The initial impetus came from an attempt to analyze the issue of stability of a black hole. Later on, the study was extended to include various types of perturbations in almost all conceivable backgrounds.

With a recent discovery of gravitational waves [3] this line of investigation became ever more important. After being perturbed, black holes return to their equilibrium by going through a ringdown phase, whose most dominant stage is characterized by the long lasting damped oscillations dubbed as quasinormal modes (QNMs) [4]. This phase is characterized by a discrete set of complex frequencies, called QNM frequencies. While the real part of the frequency corresponds to the actual frequency of the wave dynamics, the imaginary part represents the damping factor. For an overview of the subject, we refer the reader to some excellent reviews [5-8].

Several methods have been devised to determine the quasinormal modes of a black hole. They range from purely analytic methods $[9,10]$ through various semiclassical

\footnotetext{
*dmarija@ipb.ac.rs

†konjik@ipb.ac.rs

*asamsarov@irb.hr
}

Published by the American Physical Society under the terms of the Creative Commons Attribution 4.0 International license. Further distribution of this work must maintain attribution to the author(s) and the published article's title, journal citation, and DOI. Funded by SCOAP. ones [11-14] and all down to the purely numerical methods [15].

A way to describe some effects of quantum gravity is to introduce a noncommutative deformation of spacetime. The main idea of noncommutative (NC) geometry is that a spacetime as we perceive it might possibly be distorted at some relatively high energy scale, which we label as the scale of noncommutativity $l_{\mathrm{NC}}$. The scale of noncommutativity itself may be anything between the $\mathrm{TeV}$ scale and the Planck scale. Moreover, if the spacetime is indeed modified at the NC scale $l_{\mathrm{NC}}$, then this distortion should somehow be visible in the QNM spectrum of black holes. In other words, with the current capacities as well as with the new possibilities that have opened up by the detection of gravitational waves, a direct comparison of the theoretical results with the observation may signal the presence of a new physics, i.e., may hint toward a presence of an underlying NC spacetime structure above some energy threshold.

First results on NC QNM spectrum were published in $[16,17]$. There, the authors analyze the NC scalar QNM spectrum of the three-dimensional Bañados-TeitelboimZanelli (BTZ) black hole using a direct integration of the equation of motion for the NC scalar field in the BTZ black hole background.

In our recent paper [18] we have investigated a model of NC scalar and gauge fields coupled to a classical background of the Reissner-Nordström (RN) black hole. This model resulted in a master equation governing the behavior of the scalar perturbations in the presence of a noncommutative structure of spacetime. The master equation was then analyzed analytically. However, the analytic 
treatment is only possible for a highly restricted range of parameters corresponding to the near extremal case of the RN black hole. To overcome the shortcomings of the analytic treatment, in the present work we analyze the same master equation by a well-defined numerical method, the continued fraction method. This method enables us to find solutions for QNM frequencies for a more general set of system parameters. Of course, the results we obtain may also be confronted with the results obtained in [18] after specializing them to the near extremal case. Since the analytic treatment of Ref. [18] was carried out under this rather restrictive assumption, the treatment conducted here could also serve to test the validity of this assumption as well as the general accuracy of our previous analytic treatment.

To make the paper self-consistent, in the next section we repeat some of the results from our previous paper [18]. In particular, we describe the NC deformation by the angular twist and its consequences on a massive, charged scalar field propagating in the fixed (undeformed) RN background, and we derive the NC master equation for the scalar field. This equation is our starting point in Sec. III. There we investigate the QNM spectrum of the massive, charged scalar field by the Wentzel-Kramers-Brillouin (WKB) method. Our analysis is analytic, and it is therefore limited by an approximation $l+1 \ll q Q$, where $l$ is the orbital momentum of the scalar perturbation, $Q$ is the black hole charge, and $q$ is the charge of the scalar perturbation. At the end of this section, we analyze contributions of higher order WKB corrections. After this warm-up, in Sec. IV we attack the problem of finding the QNM spectrum with the well-defined numerical method, the continued fraction method. In Sec. V we discuss our results and give some final remarks. We also present our results for two realistic black hole solutions: supermassive black holes and primordial black holes. Details of a few cumbersome calculations are given in Appendixes A and B.

\section{NC SCALAR FIELD IN THE RN BACKGROUND}

A noncommutative deformation of spacetime can be introduced in different ways [19]. We follow the twist approach [20] and deform the Poincaré algebra to a twisted Poincaré algebra. The algebra relations remain the same, while the comultiplication changes. This change is relevant for multiparticle states [21]. One of the advantages of the twist deformation is that it induces a deformed differential calculus in a well-defined way. In particular, it introduces a deformed product of functions, the $\star$ product.

Our goal is to study the motion of a massive, charged scalar field in the geometry of the RN black hole described by the metric

$$
\begin{aligned}
\mathrm{d} s^{2}= & \left(1-\frac{2 M G}{r}+\frac{Q^{2} G}{r^{2}}\right) \mathrm{d} t^{2}-\frac{\mathrm{d} r^{2}}{1-\frac{2 M G}{r}+\frac{Q^{2} G}{r^{2}}} \\
& -r^{2}\left(\mathrm{~d} \theta^{2}+\sin ^{2} \theta \mathrm{d} \varphi^{2}\right) .
\end{aligned}
$$

Here $M$ is the mass of the RN black hole, while $Q$ is the charge of the RN black hole. This problem requires a model of NC gravity coupled with a NC scalar and a NC electromagnetic field. A NC deformation of gravity is not easy to construct; see [22] and references therein. Therefore, we follow a semiclassical approach based on the assumption that the geometry (gravitational field) is classical (it is not deformed by noncommutativity), while the scalar field propagating in the RN background "feels" the effects of spacetime noncommutativity. To realize this approach, we choose a Killing twist. The twist is given by

$$
\mathcal{F}=e^{-\frac{i a}{2}\left(\partial_{t} \otimes \partial_{\varphi}-\partial_{\varphi} \otimes \partial_{t}\right)} .
$$

The NC deformation is controlled by the small deformation parameter $a \approx l_{\mathrm{NC}}$. Vector fields $X_{1}=\partial_{t}, X_{2}=\partial_{\varphi}$ are commuting vector fields, $\left[X_{1}, X_{2}\right]=0$, and therefore the twist (2.2) is an Abelian twist [23]. We call (2.2) an "angular twist" because the vector field $X_{2}=\partial_{\varphi}=x \partial_{y}-$ $y \partial_{x}$ is a generator of rotations around the $z$ axis. The vector fields $X_{1}$ and $X_{2}$ are two Killing vectors for the metric (2.1) and that is why the twist (2.2) is called a Killing twist. In particular, the twist (2.2) does not act on the RN metric, and it does not act on functions of the RN metric. In this way we ensure that the geometry remains undeformed.

The twist (2.2) defines the $\star$ product of functions (fields):

$$
\begin{aligned}
f \star g & =\mu\left\{e^{\frac{i a}{2}\left(\partial_{t} \otimes \partial_{\varphi}-\partial_{\varphi} \otimes \partial_{t}\right)} f \otimes g\right\} \\
& =f g+\frac{i a}{2}\left(\partial_{t} f\left(\partial_{\varphi} g\right)-\partial_{t} g\left(\partial_{\varphi} f\right)\right)+\mathcal{O}\left(a^{2}\right) .
\end{aligned}
$$

Note that the twist (2.2) is reminiscent of the Moyal twist for the Moyal algebra $\left[x^{\star}, y\right]=i a$, where the two vector fields are $\partial_{x}$ and $\partial_{y}$, but one should refrain from introducing the star commutator $\left[t^{\star}, \varphi\right]=i a$ since $\varphi$ is not a well-defined continuous function. The following relations hold [21]:

$$
\begin{aligned}
{\left[t^{\star}, r\right] } & =0, \\
{\left[t^{\star}, e^{i \varphi}\right] } & =-a e^{i \varphi}, \\
{\left[t^{\star}, f(t, r, \theta, \varphi)\right] } & =i a \partial_{\varphi} f .
\end{aligned}
$$

One can check that the commutation relations between Decart coordinates are given by

$$
\begin{aligned}
& {\left[t^{\star}, x\right]=-i a y,} \\
& {\left[t^{\star}, y\right]=i a x .}
\end{aligned}
$$

Therefore, the twist (2.2) generates the Lie-algebra type of noncommutativity.

Using the product (2.3), one can write an action for a massive, charged scalar field in the fixed $\mathrm{RN}$ background as 


$$
S_{\phi}=\int \mathrm{d}^{4} x \sqrt{-g} \star\left(g^{\mu \nu} \star D_{\mu} \hat{\phi}^{+} \star D_{\nu} \hat{\phi}-\mu^{2} \hat{\phi}^{+} \star \hat{\phi}\right) .
$$

The scalar field $\hat{\phi}$ is a complex charged scalar field with mass $\mu$ and charge $q$. It transforms in the fundamental representation of the noncommutative $U(1)_{\star}$ gauge transformations. Therefore its covariant derivative is defined as

$$
D_{\mu} \hat{\phi}=\partial_{\mu} \hat{\phi}-i \hat{A}_{\mu} \star \hat{\phi}
$$

with NC $U(1)_{\star}$ gauge field $\hat{A}_{\mu}$. Note that $\star$ products in $\sqrt{-g} \star g^{\alpha \beta} \star g^{\mu \nu}$ can all be removed since the twist (2.2) does not act on the metric tensor (2.1).

One can check that the action (2.6) is invariant under the infinitesimal $U(1)_{\star}$ gauge transformations defined in the following way:

$$
\begin{aligned}
\delta^{\star} \hat{\phi} & =i \hat{\Lambda} \star \hat{\phi} \\
\delta^{\star} \hat{A}_{\mu} & =\partial_{\mu} \hat{\Lambda}+i\left[\hat{\Lambda} \hat{\Lambda}_{\mu}\right], \\
\delta^{\star} g_{\mu \nu} & =0
\end{aligned}
$$

with the NC gauge parameter $\hat{\Lambda}$.

Our approach is perturbative and we have to expand the action (2.6) up to first order in the deformation parameter $a$. To do that we expand the $\star$ products in (2.6) and use the Seiberg-Witten (SW) map. The SW map enables one to express NC variables as functions of the corresponding commutative variables. In this way, the problem of charge quantization in $U(1)_{\star}$ gauge theory does not exist. In the case of NC Yang-Mills theories, the SW map guarantees that the number of degrees of freedom in the NC theory is the same as in the corresponding commutative theory. That is, no new degrees of freedom are introduced.

Using the SW map, NC fields can be expressed as a function of corresponding commutative fields and can be expanded in orders of the deformation parameter $a$. Expansions for an arbitrary Abelian twist deformation are known to all orders [24]. Applying these results to the twist (2.2), expansions of fields up to first order in the deformation parameter $a$ follow. They are given by

$$
\begin{gathered}
\hat{\phi}=\phi-\frac{1}{4} \theta^{\rho \sigma} A_{\rho}\left(\partial_{\sigma} \phi+D_{\sigma} \phi\right), \\
\hat{A}_{\mu}=A_{\mu}-\frac{1}{2} \theta^{\rho \sigma} A_{\rho}\left(\partial_{\sigma} A_{\mu}+F_{\sigma \mu}\right) .
\end{gathered}
$$

The $U(1)$ covariant derivative of $\phi$ is defined as $D_{\mu} \phi=$ $\left(\partial_{\mu}-i A_{\mu}\right) \phi$, and $F_{\mu \nu}=\partial_{\mu} A_{\nu}-\partial_{\nu} A_{\mu}$ is the electromagnetic field tensor. To have more compact equations and keep track of the NC deformation, we introduce the $4 \times 4$ antisymmetric matrix $\theta^{\alpha \beta}$ with the only nonzero components $\theta^{t \varphi}=-\theta^{\varphi t}=a$. It is also important to note that the coupling constant $q$ between fields $\phi$ and $A_{\mu}$, the charge of $\phi$, is included into $A_{\mu}$, namely $A_{\mu}=q A_{\mu}$. Using the SWmap solutions and expanding the $\star$ products in (2.6) we find the action up to first order in the deformation parameter $a$. It is given by

$$
\begin{aligned}
S_{\phi}= & \int \mathrm{d}^{4} x \sqrt{-g}\left(g^{\mu \nu} D_{\mu} \phi^{+} D_{\nu} \phi-\mu^{2} \phi^{+} \phi\right. \\
& +\frac{\theta^{\alpha \beta}}{2} g^{\mu \nu}\left(-\frac{1}{2} D_{\mu} \phi^{+} F_{\alpha \beta} D_{\nu} \phi\right. \\
& \left.\left.+\left(D_{\mu} \phi^{+}\right) F_{\alpha \nu} D_{\beta} \phi+\left(D_{\beta} \phi^{+}\right) F_{\alpha \mu} D_{\nu} \phi\right)\right) .
\end{aligned}
$$

The equation of motion for the field $\phi$ is obtained by varying this action with respect to $\phi$ and it is given by

$$
\begin{aligned}
g^{\mu \nu} & \left(\left(\partial_{\mu}-i A_{\mu}\right) D_{\nu} \phi-\Gamma_{\mu \nu}^{\lambda} D_{\lambda} \phi\right) \\
& -\frac{1}{4} \theta^{\alpha \beta} g^{\mu \nu}\left(\left(\partial_{\mu}-i A_{\mu}\right)\left(F_{\alpha \beta} D_{\nu} \phi\right)-\Gamma_{\mu \nu}^{\lambda} F_{\alpha \beta} D_{\lambda} \phi\right. \\
& -2\left(\partial_{\mu}-i A_{\mu}\right)\left(F_{\alpha \nu} D_{\beta} \phi\right)+2 \Gamma_{\mu \nu}^{\lambda} F_{\alpha \lambda} D_{\beta} \phi \\
& \left.-2\left(\partial_{\beta}-i A_{\beta}\right)\left(F_{\alpha \mu} D_{\nu} \phi\right)\right)=0,
\end{aligned}
$$

with $\Gamma_{\mu \nu}^{\lambda}$ being the Christoffel symbols corresponding to the metric (2.1). The RN background also fixes the $U(1)$ gauge field $A_{\mu}$ to

$$
A_{0}=-\frac{q Q}{r}
$$

This is the electromagnetic potential of the pointlike charge $Q$ located at $r=0$. Consequently, the only nonzero component of the field strength tensor $F_{\mu \nu}$ is the radial electric field

$$
F_{r 0}=\frac{q Q}{r^{2}} .
$$

Furthermore, since the only nonzero components of the NC deformation parameter $\theta^{\alpha \beta}$ are $\theta^{t \varphi}=-\theta^{\varphi t}=a$, the equation of motion (2.11) simplifies to

$$
\begin{gathered}
\left(\frac{1}{f} \partial_{t}^{2}-\Delta+(1-f) \partial_{r}^{2}+\frac{2 M G}{r^{2}} \partial_{r}+2 i q Q \frac{1}{r f} \partial_{t}-\frac{q^{2} Q^{2}}{r^{2} f}\right) \phi \\
+\frac{a q Q}{r^{3}}\left(\left(\frac{M G}{r}-\frac{G Q^{2}}{r^{2}}\right) \partial_{\varphi}+r f \partial_{r} \partial_{\varphi}\right) \phi=0
\end{gathered}
$$

where $\Delta$ is the usual Laplace operator. We also introduced

$$
f=1-\frac{2 M G}{r}+\frac{Q^{2} G}{r^{2}}=\frac{\left(r-r_{+}\right)\left(r-r_{-}\right)}{r^{2}}
$$

with $r_{ \pm}=M G \pm \sqrt{M^{2} G^{2}-Q^{2} G}$. To simplify the notation, we will set $G=1$ in the following. 
To solve this equation we assume an ansatz [18]

$$
\phi_{l m}(t, r, \theta, \varphi)=R_{l m}(r) e^{-i \omega t} Y_{l}^{m}(\theta, \varphi)
$$

with spherical harmonics $Y_{l}^{m}(\theta, \varphi)$. Inserting (2.16) into (2.14) leads to an equation for the radial function $R_{l m}(r)$,

$$
\begin{aligned}
f R_{l m}^{\prime \prime} & +\frac{2}{r}\left(1-\frac{M}{r}\right) R_{l m}^{\prime} \\
& -\left(\frac{l(l+1)}{r^{2}}-\frac{1}{f}\left(\omega-\frac{q Q}{r}\right)^{2}+\mu^{2}\right) R_{l m} \\
& -i m a \frac{q Q}{r^{3}}\left(\left(\frac{M}{r}-\frac{Q^{2}}{r^{2}}\right) R_{l m}+r f R_{l m}^{\prime}\right)=0 .
\end{aligned}
$$

We note that the first line of this equation, which describes the system without deformation, corresponds to the equation for the radial function $R_{l m}$ analyzed in [25,26]. The NC contribution in (2.17) vanishes for a neutral scalar field, that is for $q=0$, while it does not depend on the scalar field mass $\mu$.

\section{QNM SPECTRUM: WKB ANALYSIS}

Quasinormal modes are a particular solution of Eq. (2.17). They are specified by the following boundary conditions: purely incoming at the horizon and purely outgoing in the infinity. We mentioned in the Introduction that there are different ways to solve Eq. (2.17) and find the corresponding QNM spectrum. To warm up, we start with the WKB approach and present an analytic solution for the QNM frequencies, valid for a specific range of parameters. Later on, in Sec. IV we move to a more general numerical method, the continued fraction method.

\section{A. A modified tortoise coordinate}

The starting point of the WKB method is a Schrödinger type equation:

$$
\frac{\mathrm{d}^{2} \psi}{\mathrm{d} r_{*}^{2}}+V \psi=0
$$

Therefore, we have to transform Eq. (2.17) into this form. To start with, we have to define the tortoise coordinate $r_{*}$. However, the usual definition of $r_{*}$,

$$
\mathrm{d} r_{*}=\frac{\mathrm{d} r}{f}
$$

does not lead to an equation of the type (3.1). The form of the equation is spoiled by the presence of terms that are linear in $\frac{\mathrm{d} \psi}{\mathrm{d} r_{*}}$. The origin of these terms is the presence of the deformation parameter $a$. Fortunately, it is possible to circumvent this problem by conveniently extending the definition of the tortoise coordinate. Indeed, one can show that the following change of coordinates

$$
\mathrm{d} y=\frac{\mathrm{d} r}{f\left(1+i a m \frac{q Q}{r}\right)}
$$

brings Eq. (2.17) into the form

$$
\frac{\mathrm{d}^{2} \psi}{\mathrm{d} y^{2}}+V \psi=0
$$

where $\psi=r R$ and

$$
\begin{aligned}
V= & r f\left[-\frac{2}{r^{3}}\left(\frac{M}{r}-\frac{Q^{2}}{r^{2}}\right)-\frac{1}{r}\left(\frac{l(l+1)}{r^{2}}-\frac{1}{f}\left(\omega-\frac{q Q}{r}\right)^{2}+\mu^{2}\right)\right. \\
& \left.+i a m \frac{q Q}{r^{4}}\left(1-\frac{7 M}{r}+6 \frac{Q^{2}}{r^{2}}\right)-2 i a m \frac{q Q}{r^{2}}\left(\frac{l(l+1)}{r^{2}}-\frac{1}{f}\left(\omega-\frac{q Q}{r}\right)^{2}+\mu^{2}\right)\right] .
\end{aligned}
$$

Note that, as in all our calculations, this equation is valid up to first order in the deformation parameter $a$.

It is interesting to see what is the explicit form of the tortoise coordinate defined by (3.3). To see this, we note that up to first order in $a$ the relation (3.3) can equivalently be written as

$$
y=y^{(0)}+y^{(1)}=\int \frac{\mathrm{d} r}{f}-i a m q Q \int \frac{\mathrm{d} r}{r f},
$$

clearly separating a required transformation into two parts. The first part is the standard Reissner-Nordström tortoise coordinate $y^{(0)}=r_{*}^{\mathrm{RN}}$ while the second part represents the term coming exclusively from the NC deformation.

Integrating (3.6) we find

$$
\begin{aligned}
y & =y^{(0)}-\operatorname{iamq} Q\left\{\frac{r_{+}}{r_{+}-r_{-}} \ln \left(r-r_{+}\right)-\frac{r_{-}}{r_{+}-r_{-}} \ln \left(r-r_{-}\right)\right\} \\
& =r+\frac{r_{+}}{r_{+}-r_{-}}\left(r_{+}-i a m q Q\right) \ln \left(r-r_{+}\right)-\frac{r_{-}}{r_{+}-r_{-}}\left(r_{-}-\operatorname{iamq} Q\right) \ln \left(r-r_{-}\right),
\end{aligned}
$$


where $y^{(0)}$ is the standard tortoise coordinate for the Reissner-Nordström metric given by

$y^{(0)} \equiv r_{*}^{\mathrm{RN}}=r+\frac{r_{+}^{2}}{r_{+}-r_{-}} \ln \left(r-r_{+}\right)-\frac{r_{-}^{2}}{r_{+}-r_{-}} \ln \left(r-r_{-}\right)$.

We repeat once again that the result (3.7) is valid up to first order in the parameter $a$. The corrections in (3.7) are such that they do not change the position of the event horizons, which coincides with the analysis of the Hawking radiation in a semiclassical tunneling formalism [18,27].

The coordinate $y$ (3.7) has the standard properties of a tortoise coordinate. As $r \rightarrow+\infty, y \rightarrow+\infty$, and as $r \rightarrow r_{+}$, $y \rightarrow-\infty$. Moreover, a brief inspection of the potential (3.5) shows that as $r \rightarrow+\infty$, the potential $V$ tends to $V \rightarrow$ $\omega^{2}-\mu^{2}$. Similarly, as $r \rightarrow r_{+}$, the potential $V$ approaches the value $V \rightarrow\left(\omega-\frac{q Q}{r_{+}}\right)^{2}\left(1-2 i a m \frac{q Q}{r_{+}}\right)$. These two limiting values completely agree with the behavior of the effective potential found in [28] for the massive charged scalar field in the Kerr-Newman background (after putting the black hole angular momentum of Ref. [28] to zero and taking the commutative limit of our result).

\section{B. QNM spectrum}

The WKB method is based on the similarity between the equation governing the behavior of a black hole perturbation (scalar in our case) and the Schrödinger equation in the case of a potential barrier [11-13]. The condition for QNMs is obtained by matching two WKB solutions on each side of the potential barrier given by $-V$ to the solution inside the barrier, with the matching done simultaneously across both of the turning points. The matching procedure [8] leads to the following condition for the QNM frequencies:

$$
-\frac{V_{0}}{\sqrt{2 V_{0}^{\prime \prime}}}-i \sum_{j=2}^{6} \Lambda_{j}=i\left(n+\frac{1}{2}\right)
$$

where $n=0,1,2,3, \ldots$ This condition clearly involves finding an extremal value of the potential $V$ and the value of the curvature at the extremal point $[11,12]$. In addition, the correction terms $\Lambda_{j}$ depend on the value of the effective potential and its derivatives (up to $j$ th order) in the maximum. The explicit form of the WKB corrections $\Lambda_{2}$ and $\Lambda_{3}$ can be found in [12] and of $\Lambda_{4}, \Lambda_{5}, \Lambda_{6}$ in [29]. Because of the additional terms in (3.5) induced by noncommutativity, the higher WKB corrections might be very cumbersome to calculate. To arrive at a result for QNM frequencies that can be written in a closed analytic form, at this point we purposefully restrict to only a most dominating term in the quantization condition (3.9), thus ignoring the higher $\Lambda$ terms. By doing this we assume that the higher order corrections are small enough, so that they do not interfere with $\mathrm{NC}$ contributions coming from the first term in (3.9). In particular, we expect that this assumption may be true especially in the limit of large angular momentum $l$. Whether this assumption is true or not is a question that we shall address later. For now, let us focus on the most dominant term in (3.9) and postpone the analysis of the higher order WKB corrections for the very end of this section.

To proceed we follow [26] and introduce new dimensionless variables

$$
x=\frac{r-r_{+}}{r}, \quad \Omega=\frac{\omega r_{+}}{q Q}-1 .
$$

In terms of these variables the effective potential can be written as

$$
\begin{aligned}
V(x)= & -V_{\mathrm{eff}}=\left(\frac{q Q}{r_{+}}\right)^{2}(x+\Omega)^{2}-\frac{r_{+}-r_{-}}{r_{+}}\left(\mu^{2}+\frac{H\left(r_{+}\right)}{r_{+}^{2}}\right) x \\
& +i a m \frac{q Q}{r_{+}} \frac{r_{+}-r_{-}}{r_{+}}\left(\frac{1}{r_{+}^{2}}-\frac{7 M}{r_{+}^{3}}+6 \frac{Q^{2}}{r_{+}^{4}}-2 \frac{l(l+1)}{r_{+}^{2}}-2 \mu^{2}\right) x \\
& +4 i a m\left(\frac{q Q}{r_{+}}\right)^{3} \Omega x+2 \operatorname{iam}\left(\frac{q Q}{r_{+}}\right)^{3} \Omega^{2}(1-x)+O\left(x^{2}, a x^{2}\right),
\end{aligned}
$$

where $\quad H\left(r_{+}\right)=l(l+1)+\frac{2 M}{r_{+}}-\frac{2 Q^{2}}{r_{+}^{2}}=l(l+1)+\frac{r_{+}-r_{-}}{r_{+}}$. It is obvious from these definitions that $H\left(r_{+}\right)<$ $l(l+1)+1$. Note that we discarded all terms quadratic in $x$ other than the first parabolic term $\sim(x+\Omega)^{2}$ as nonphysical. Indeed, if we keep all quadratic terms, the resulting effective potential does not describe the realistic problem at hand. In particular, within our approximation the parabolic term $\sim(x+\Omega)^{2}$ has a peak immediately next to the horizon. Retaining other terms quadratic in $x$ would only move the peak of the potential far away from the event horizon, and this is physically undesirable. That is why, of all terms quadratic in $x$, we keep only the first parabolic term $\sim(x+\Omega)^{2}$ in the effective potential. Moreover, retaining terms of the order $O\left(a x^{2}\right)$ in the potential function would only produce corrections to the position of its extremal point that are of the second order within our approximation and thus can be neglected.

As far as the effective potential of the ReissnerNordström black hole due to charged scalar perturbations is concerned, the analysis carried out in Ref. [26] has been 
focused on the regime $q Q \gg l+1$ with a purpose of being able to deal with the condition (3.9) analytically in that particular case. For the same reason we also restrict our analysis to this regime. Putting this together with the just established relation $H\left(r_{+}\right)<l(l+1)+1$, it is clear that this condition translates into $\frac{H\left(r_{+}\right)}{q^{2} Q^{2}} \ll 1$. In addition, we also assume that $\frac{\mu^{2} r_{+}^{2}}{q^{2} Q^{2}} \ll 1$.

It is reasonable to expect the QNM frequencies to be centered around the classical result $\omega=\frac{q Q}{r_{+}}$. Therefore, it is natural to assume that the quantity $\Omega$ is also very small.
As in Ref. [26], we assume here that it is of the same order as $\frac{H\left(r_{+}\right)}{q^{2} Q^{2}}$ and $\frac{\mu^{2} r_{+}^{2}}{q^{2} Q^{2}}$. Furthermore, our analysis includes a small $\mathrm{NC}$ parameter $a$, and we assume that it is also of the same order as the former small quantities. To be more precise, we have to assume that $l a \frac{q Q}{r_{+}} \ll 1$, where $l$ is the orbital momentum of the perturbation. To summarize, our approximation can be written as $\frac{H\left(r_{+}\right)}{q^{2} Q^{2}} \sim \frac{\mu^{2} r_{+}^{2}}{q^{2} Q^{2}} \sim \Omega \sim l a \frac{q Q}{r_{+}} \ll 1$.

The position of the extremum of the effective potential is determined by the condition $\left.\frac{\mathrm{d} V}{\mathrm{~d} x}\right|_{x_{0}}=0$, leading to

$$
\begin{aligned}
x_{0}+\Omega= & \frac{1}{2 q^{2} Q^{2}} \frac{r_{+}-r_{-}}{r_{+}}\left[H\left(r_{+}\right)+\mu^{2} r_{+}^{2}-i a m \frac{q Q}{r_{+}}\left(1-\frac{7 M}{r_{+}}+\frac{6 Q^{2}}{r_{+}^{2}}-2 l(l+1)-2 \mu^{2} r_{+}^{2}\right)\right] \\
& -2 i a m \frac{q^{3} Q^{3}}{r_{+}}\left(2 \Omega-\Omega^{2}\right) .
\end{aligned}
$$

It is easily seen that within our approximation, the value of $x_{0}=\frac{r_{0}-r_{+}}{r_{0}}$ is very small, ensuring that the peak of the potential is close to the event horizon, as it should be. The value of $r_{0}$ can be obtained from the relation $x_{0}=1-\frac{r_{+}}{r_{0}}$, leading to

$$
\begin{aligned}
\frac{1}{r_{0}}= & \frac{\Omega+1}{r_{+}}-\frac{1}{2 q^{2} Q^{2}} \frac{r_{+}-r_{-}}{r_{+}^{2}}\left[H\left(r_{+}\right)+\mu^{2} r_{+}^{2}-i a m \frac{q Q}{r_{+}}\left(1-\frac{7 M}{r_{+}}+\frac{6 Q^{2}}{r_{+}^{2}}-2 l(l+1)-2 \mu^{2} r_{+}^{2}\right)\right] \\
& +2 i a m \frac{q^{3} Q^{3}}{r_{+}^{2}}\left(2 \Omega-\Omega^{2}\right) .
\end{aligned}
$$

Up to first order in $\frac{H\left(r_{+}\right)}{q^{2} Q^{2}}, \frac{\mu^{2} r_{+}^{2}}{q^{2} Q^{2}}, \Omega$, and $l a \frac{q Q}{r_{+}}$this equation can be inverted to give

$$
r_{0}=r_{+}\left[1-\Omega+\left(r_{+}-r_{-}\right)\left[\frac{H\left(r_{+}\right)}{2 q^{2} Q^{2} r_{+}}+\frac{\mu^{2} r_{+}}{2 q^{2} Q^{2}}-i a m \frac{1}{2 q Q r_{+}^{2}}\left(1-\frac{7 M}{r_{+}}+\frac{6 Q^{2}}{r_{+}^{2}}-2 l(l+1)-2 \mu^{2} r_{+}^{2}\right)\right]\right] .
$$

In the same way one finds the remaining quantities that will be required in the analysis; see the relation (3.15):

$$
\left(\frac{\mathrm{d} x}{\mathrm{~d} r}\right)_{r=r_{0}}=\frac{1}{r_{+}}\left[1+2 \Omega-\frac{1}{q^{2} Q^{2}} \frac{r_{+}-r_{-}}{r_{+}}\left[H\left(r_{+}\right)+\mu^{2} r_{+}^{2}-i a m \frac{q Q}{r_{+}}\left(1-\frac{7 M}{r_{+}}+\frac{6 Q^{2}}{r_{+}^{2}}-2 l(l+1)-2 \mu^{2} r_{+}^{2}\right)\right]\right]
$$

and

$$
\begin{aligned}
f\left(r_{0}\right) & =\frac{\left(r_{0}-r_{+}\right)\left(r_{0}-r_{-}\right)}{r_{0}^{2}} \\
& =\frac{r_{+}-r_{-}}{r_{+}^{2}}\left[r_{+}\left(r_{+}-r_{-}\right)\left[\frac{H\left(r_{+}\right)}{2 q^{2} Q^{2} r_{+}}+\frac{\mu^{2} r_{+}}{2 q^{2} Q^{2}}-i a m \frac{1}{2 q Q r_{+}^{2}}\left(1-\frac{7 M}{r_{+}}+\frac{6 Q^{2}}{r_{+}^{2}}-2 l(l+1)-2 \mu^{2} r_{+}^{2}\right)\right]-\Omega r_{+}\right] .
\end{aligned}
$$

The extremal value $V_{0}$ of the negative effective potential $V(x)=-V_{\text {eff }}$ is

$$
V_{0}=-\frac{1}{4 q^{2} Q^{2} r_{+}^{2}}\left(\frac{r_{+}-r_{-}}{r_{+}}\right)^{2}\left(H\left(r_{+}\right)+\mu^{2} r_{+}^{2}\right)^{2}+\mu^{2} \Omega \frac{r_{+}-r_{-}}{r_{+}}+\frac{H\left(r_{+}\right)\left(r_{+}-r_{-}\right)}{r_{+}^{3}} \Omega
$$

Once again, note that all these quantities have been written up to only first order in $\frac{H}{q^{2} Q^{2}}, \frac{\mu^{2}}{q^{2} Q^{2}}, \Omega$, and $l a \frac{q Q}{r_{+}}$. 
Since at the extremal point $x_{0}$ the first derivative $\frac{\mathrm{d} V}{\mathrm{~d} x}$ vanishes, the QNM condition (3.9) implies

$$
-V_{0}=i\left(n+\frac{1}{2}\right) \sqrt{2\left(\frac{\mathrm{d}^{2} V}{\mathrm{~d} x^{2}}\right)_{x=x_{0}}\left(\frac{\mathrm{d} x}{\mathrm{~d} r}\right)_{r=r_{0}}^{2}\left(\frac{\mathrm{d} r}{\mathrm{~d} y}\right)_{y=y_{0}}^{2}} .
$$

Knowing that $\frac{\mathrm{d} r}{\mathrm{~d} y}=f(r)\left(1+i a m \frac{q Q}{r}\right)$ and inserting the above expressions for $\left(\frac{\mathrm{d} x}{\mathrm{~d} r}\right)_{r=r_{0}}, V_{0}$, and $f\left(r_{0}\right)$ into (3.15) lead to a coupled system of equations,

$$
\begin{aligned}
& \frac{\left(r_{+}-r_{-}\right)}{4 q^{2} Q^{2} r_{+}}\left(H\left(r_{+}\right)+\mu^{2} r_{+}^{2}\right)^{2}-\Omega_{R}\left[H\left(r_{+}\right)+\mu^{2} r_{+}^{2}\right] \\
& =2 q Q\left(n+\frac{1}{2}\right) \Omega_{I}+a m\left(n+\frac{1}{2}\right) \frac{r_{+}-r_{-}}{r_{+}^{2}}\left(1-\frac{7 M}{r_{+}}+\frac{6 Q^{2}}{r_{+}^{2}}-2 l(l+1)-2 \mu^{2} r_{+}^{2}\right), \\
& \quad-\Omega_{I}\left[H\left(r_{+}\right)+\mu^{2} r_{+}^{2}\right]=\left(n+\frac{1}{2}\right) \frac{r_{+}-r_{-}}{q Q r_{+}}\left[H\left(r_{+}\right)+\mu^{2} r_{+}^{2}\right]-2 q Q\left(n+\frac{1}{2}\right) \Omega_{R},
\end{aligned}
$$

where $\Omega$ has been split into its real and imaginary parts, $\Omega=\Omega_{R}+i \Omega_{I}$. The solution of this system of equations is given by

$$
\begin{aligned}
\Omega_{R}= & \frac{\left(r_{+}-r_{-}\right)\left(H\left(r_{+}\right)+\mu^{2} r_{+}^{2}\right)}{r_{+}\left(\left(H\left(r_{+}\right)+\mu^{2} r_{+}^{2}\right)^{2}+4 q^{2} Q^{2}\left(n+\frac{1}{2}\right)^{2}\right)} \\
& \times\left[\frac{\left(H\left(r_{+}\right)+\mu^{2} r_{+}^{2}\right)^{2}}{4 q^{2} Q^{2}}+2\left(n+\frac{1}{2}\right)^{2}-\frac{a m}{r_{+}}\left(n+\frac{1}{2}\right)\left(1-\frac{7 M}{r_{+}}+\frac{6 Q^{2}}{r_{+}^{2}}-2 l(l+1)-2 \mu^{2} r_{+}^{2}\right)\right], \\
\Omega_{I} & =\frac{\left(r_{+}-r_{-}\right)}{2 q Q r_{+}}\left[-\frac{\left(H\left(r_{+}\right)+\mu^{2} r_{+}^{2}\right)^{2}}{4 q^{2} Q^{2}}-\frac{a m}{r_{+}}\left(n+\frac{1}{2}\right)\left(1-\frac{7 M}{r_{+}}+\frac{6 Q^{2}}{r_{+}^{2}}-2 l(l+1)-2 \mu^{2} r_{+}^{2}\right)\right] .
\end{aligned}
$$

Using (3.10) one can express the real and the imaginary parts of the QNM frequency $\omega$. For completeness, in Fig. 1 we plot the dependence of $\operatorname{Re} \omega$ and $\operatorname{Im} \omega$ on $q Q$ in the case of $\mu=0.05, l=100$, and $a=0.000001$. It is obvious from Eq. (3.19) that there will be a splitting of frequencies for different values of the projection of angular momentum $m$. This result is in agreement with our previous findings in [18]. The frequency splittings $\omega^{ \pm}=\omega(m= \pm 100)-$ $\omega(m=0)$ are plotted in Fig. 2.

The straightforward comparison of the results obtained by the WKB method with our previous results in [18]

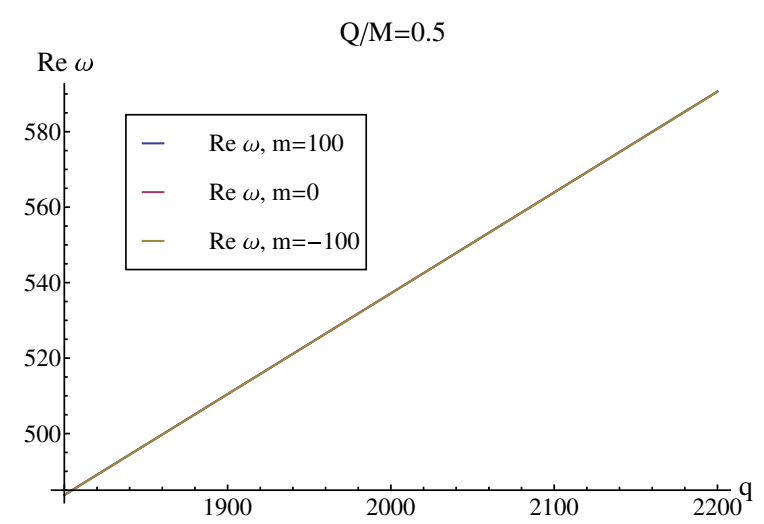

obtained analytically in the near-extremal limit is not possible. A well-known feature of the WKB method is that it gives better results for higher values of the angular momentum $l$. Therefore we plotted our results for the case $l=100$. On the other hand, in [18] we analyzed only $l=1$ and $l=2$ cases. However, the qualitative comparison shows the frequency splitting due to noncommutativity in both cases and confirms our conclusions about the effects of noncommutative deformation on the QNM spectrum. Notice that, unlike in [18], the splitting in $\operatorname{Im} \omega$ is already visible in Fig. 1. Namely, we plotted three cases $m=-100, m=0$,

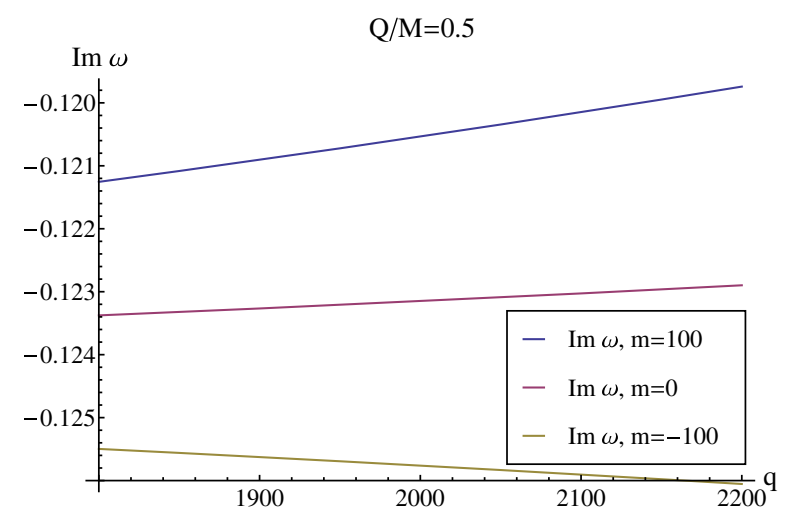

FIG. 1. Dependence of $\operatorname{Re} \omega$ (left) and $\operatorname{Im} \omega$ (right) on the charge $q Q$ of the scalar field with the mass $\mu=0.05$ and the orbital momentum $l=100$. 

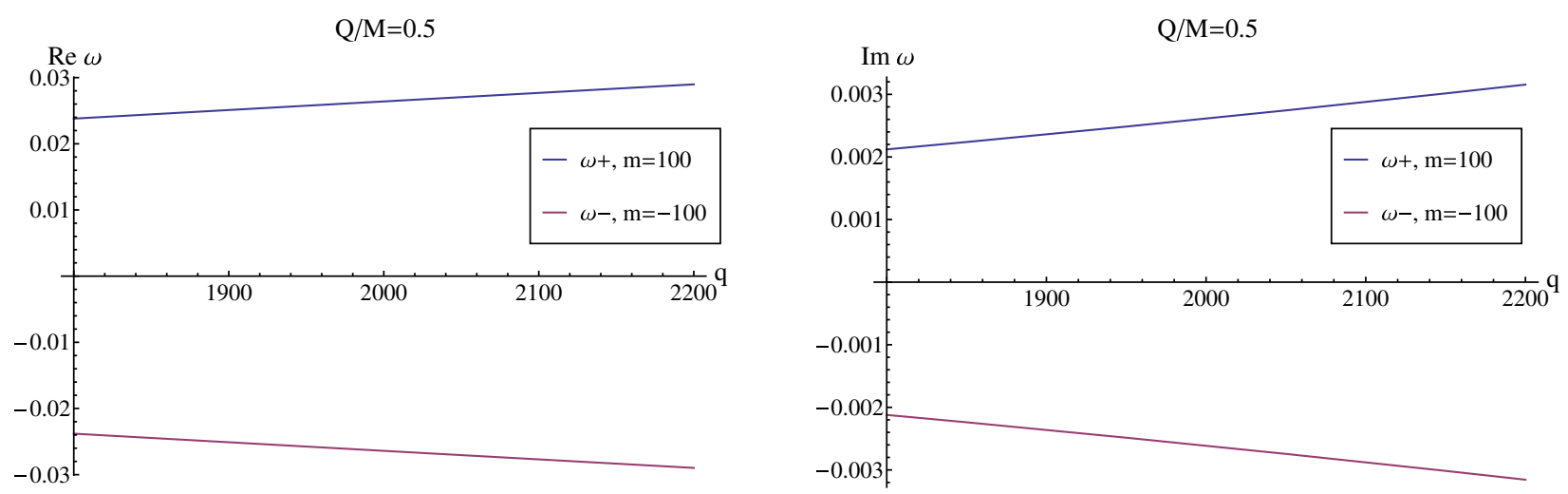

FIG. 2. Dependence of $\operatorname{Re} \omega^{ \pm}$(left) and $\operatorname{Im} \omega^{ \pm}$(right) on the charge $q Q$ of the scalar field with the mass $\mu=0.05$ and $m= \pm 100$.

and $m=100$. Since the NC corrections are proportional to $a m$, it is obvious that the effect of noncommutativity will be larger for bigger $m$. The relative splitting can be estimated from Figs. $1-4$ as $\delta_{\mathrm{Re}} \sim \frac{\mathrm{Re} \omega^{+}}{\mathrm{Re} \omega} \sim 10^{-4}$. In a similar way, for the imaginary part we estimate $\delta_{\operatorname{Im}} \sim \frac{\operatorname{Im} \omega^{+}}{\operatorname{Im} \omega} \sim 10^{-2}$. The splitting is obviously bigger than the splitting found in [18], and this enhancement is due to the high angular momentum $l=100$.

Finally, to compare our results with the commutative results obtained in [26], we have to make one additional approximation. Namely, we assume that $\frac{q^{2} Q^{2}}{\left(H\left(r_{+}\right)+\mu^{2} r_{+}^{2}\right)^{2}} \ll 1$, and at the same time being of the same order of magnitude as $\frac{H\left(r_{+}\right)}{q^{2} Q^{2}}, \frac{\mu^{2} r_{+}^{2}}{q^{2} Q^{2}}, \Omega$, and $a l \frac{q Q}{r_{+}}$. This final approximation amounts to requiring $q^{2} Q^{2} \ll l^{4}$. Taken together, our approximations select the parameter range to be $l \ll$ $q Q \ll l^{2}$.

Solving the above system of equations for $\Omega_{R}$ and $\Omega_{I}$ then leads, respectively, to

$$
\begin{aligned}
\Omega_{R}= & \frac{r_{+}-r_{-}}{4 q^{2} Q^{2} r_{+}}\left(H\left(r_{+}\right)+\mu^{2} r_{+}^{2}\right)\left[1+\frac{8 q^{2} Q^{2}}{\left(H\left(r_{+}\right)+\mu^{2} r_{+}^{2}\right)^{2}}\left(n+\frac{1}{2}\right)^{2}\right. \\
& \left.-\frac{4 q^{2} Q^{2}}{r_{+}\left(H\left(r_{+}\right)+\mu^{2} r_{+}^{2}\right)^{2}} \operatorname{am}\left(n+\frac{1}{2}\right)\left(1-\frac{7 M}{r_{+}}+\frac{6 Q^{2}}{r_{+}^{2}}-2 l(l+1)-2 \mu^{2} r_{+}^{2}\right)\right] \\
\approx & \frac{r_{+}-r_{-}}{4 q^{2} Q^{2} r_{+}}\left(H\left(r_{+}\right)+\mu^{2} r_{+}^{2}\right)\left[1+\frac{8 q^{2} Q^{2}}{\left(H\left(r_{+}\right)+\mu^{2} r_{+}^{2}\right)^{2}}\left(n+\frac{1}{2}\right)^{2}\right]
\end{aligned}
$$

and

$$
\begin{aligned}
\Omega_{I}= & -\frac{r_{+}-r_{-}}{q Q r_{+}}\left(n+\frac{1}{2}\right)+\frac{1}{\left(H\left(r_{+}\right)+\mu^{2} r_{+}^{2}\right)^{2}} \frac{r_{+}-r_{-}}{r_{+}}\left(n+\frac{1}{2}\right) \\
& \times\left[\frac{1}{2 q Q}\left(H\left(r_{+}\right)+\mu^{2} r_{+}^{2}\right)^{2}+4 q Q\left(n+\frac{1}{2}\right)^{2}-2 a m \frac{q Q}{r_{+}}\left(n+\frac{1}{2}\right)\left(1-\frac{7 M}{r_{+}}+\frac{6 Q^{2}}{r_{+}^{2}}-2 l(l+1)-2 \mu^{2} r_{+}^{2}\right)\right] \\
\approx & -\frac{r_{+}-r_{-}}{q Q r_{+}}\left(n+\frac{1}{2}\right)+\frac{1}{\left(H\left(r_{+}\right)+\mu^{2} r_{+}^{2}\right)^{2}} \frac{r_{+}-r_{-}}{r_{+}}\left(n+\frac{1}{2}\right)\left[\frac{1}{2 q Q}\left(H\left(r_{+}\right)+\mu^{2} r_{+}^{2}\right)^{2}+4 q Q\left(n+\frac{1}{2}\right)^{2}\right] .
\end{aligned}
$$

Within this approximation, there is no contribution of noncommutativity in both $\Omega_{R}$ and $\Omega_{I}$. The terms in the second line in (3.20) and (3.21) are second order "small" being a product " of $\frac{q^{2} Q^{2}}{\left(H\left(r_{+}\right)+\mu^{2} r_{+}^{2}\right)^{2}}$ and $a m \frac{1}{r_{+}}$in (3.20) and $\frac{1}{\left(H\left(r_{+}\right)+\mu^{2} r_{+}^{2}\right)^{2}}$ and $a m \frac{q Q}{r_{+}}$in (3.21). To compare the obtained results with the results in [26], we have to set the mass of the field $\mu=0$ and the noncommutativity parameter $a=0$. After this, we indeed find the agreement with (19) and (20) in [26]. However, the results in [26] are zeroth order in small variables $\frac{q^{2} Q^{2}}{H^{2}}, \frac{H}{q^{2} Q^{2}}, \Omega$, while our results also contain the first order corrections in these variables.

\footnotetext{
${ }^{1}$ Note that $q Q$ is always bigger than 1 , since $l \ll q Q \ll l^{2}$. According to our approximation $a l \frac{q Q}{r_{+}} \ll 1$; therefore $a l \frac{1}{r_{+}} \ll 1$ is also valid. In the same way $\frac{q^{2} Q^{2}}{\left(H\left(r_{+}\right)+\mu^{2} r_{+}^{2}\right)^{2}} \ll 1$ implies $\frac{1}{\left(H\left(r_{+}\right)+\mu^{2} r_{+}^{2}\right)^{2}} \ll 1$.
} 


\section{Higher order WKB corrections}

As indicated earlier, we now return to the quantization condition (3.9) and reconsider it by taking into account the higher order WKB terms. In particular, we include in a calculation the higher WKB correction terms $\Lambda_{2}$ and $\Lambda_{3}$ appearing in the quantization condition (3.9). The corrections $\Lambda_{2}$ and $\Lambda_{3}$ are given as

$$
\begin{aligned}
\Lambda_{2}= & \frac{1}{\sqrt{2 V_{0}^{\prime \prime}}}\left[\frac{1}{8} \frac{V_{0}^{(4)}}{V_{0}^{\prime \prime}}\left(\frac{1}{4}+\left(n+\frac{1}{2}\right)^{2}\right)\right. \\
& \left.-\frac{1}{288}\left(\frac{V_{0}^{(3)}}{V_{0}^{\prime \prime}}\right)^{2}\left(7+60\left(n+\frac{1}{2}\right)^{2}\right)\right], \\
\Lambda_{3}= & \frac{n+\frac{1}{2}}{2 V_{0}^{\prime \prime}}\left[\frac{5}{6912}\left(\frac{V_{0}^{(3)}}{V_{0}^{\prime \prime}}\right)^{4}\left(77+188\left(n+\frac{1}{2}\right)^{2}\right)\right. \\
& -\frac{1}{384} \frac{\left(V_{0}^{(3)}\right)^{2} V_{0}^{(4)}}{\left(V_{0}^{\prime \prime}\right)^{3}}\left(51+100\left(n+\frac{1}{2}\right)^{2}\right) \\
& +\frac{1}{2304}\left(\frac{V_{0}^{(4)}}{V_{0}^{\prime \prime}}\right)^{2}\left(67+68\left(n+\frac{1}{2}\right)^{2}\right) \\
& +\frac{1}{288} \frac{V_{0}^{(3)} V_{0}^{(5)}}{\left(V_{0}^{\prime \prime}\right)^{2}}\left(19+28\left(n+\frac{1}{2}\right)^{2}\right) \\
& \left.-\frac{1}{288} \frac{V_{0}^{(6)}}{V_{0}^{\prime \prime}}\left(5+4\left(n+\frac{1}{2}\right)^{2}\right)\right] .
\end{aligned}
$$

Here $V$ is the potential (3.5) and accordingly, $V_{0}$ is a peak value of $V$; that is, the value of $V$ at its extremal point $r=r_{0}$. Analogously, $V_{0}^{\prime \prime}=\left(\mathrm{d}^{2} V / \mathrm{d} y^{2}\right)_{r=r_{0}}$ and $V_{0}^{(n)}=$ $\left(\mathrm{d}^{n} V / \mathrm{d} y^{n}\right)_{r=r_{0}}, n>2$ with $y$ being the tortoise coordinate (3.7). The fact that $V$ is expressed in terms of the variable $r$, while at the same time the expressions for $\Lambda_{2}$ and $\Lambda_{3}$ include derivatives with respect to the tortoise coordinate $y$ may complicate a calculation to some extent, since the straightforward application of the chain rule for derivation results in very cumbersome expressions.

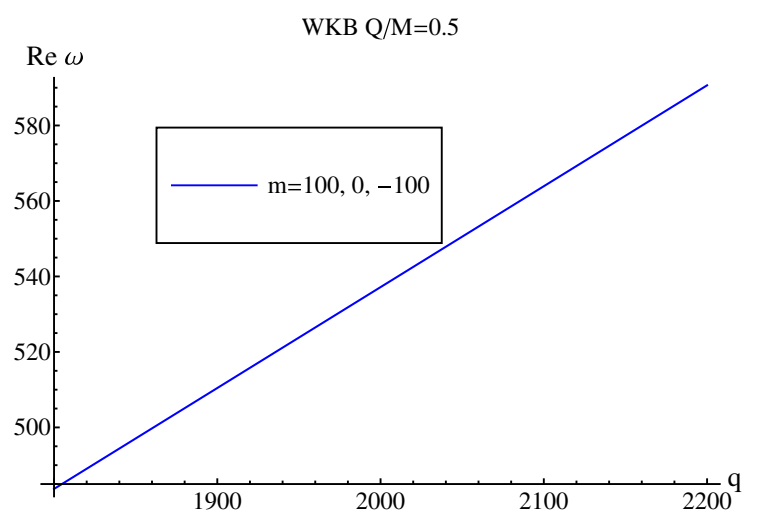

Before presenting the improved results for the QNM frequencies, it is worthwhile to estimate the order of magnitude of the corrections $\Lambda_{2}$ and $\Lambda_{3}$, as compared to the most dominant contribution in (3.9), which is the only one that has been retained in the initial approximation. This will give us feedback as to whether the introduction of $\Lambda_{2}$ and $\Lambda_{3}$ into an analysis is legitimate. For that purpose we fix the system parameters as before, $l=100, m=100$, $n=0, \mu=0.05, q=1800$ (so that $q Q=900$ ), $M=1$, $a=10^{-6}$ (see Figs. 1 and 2), and analyze the following ratios:

$$
\begin{aligned}
& \frac{\Lambda_{2}}{V_{0} / \sqrt{2 V_{0}^{\prime \prime}}} \sim 8 \times 10^{-3}-2.5 \times 10^{-6} i \\
& \frac{\Lambda_{3}}{V_{0} / \sqrt{2 V_{0}^{\prime \prime}}} \sim 4.6 \times 10^{-8}+4.4 \times 10^{-9} i .
\end{aligned}
$$

Given that a typical NC term in the real as well as in the imaginary part of the potential $V$ is of the form $a l \frac{q Q}{r_{+}}$and that the system parameters are fixed as above, we find that in the real and imaginary part of $\omega$ the noncommutative terms give rise to contributions of the order $10^{-4}$. This makes them comparable with $\Lambda_{2}$ to a reasonable extent. However, unlike $\Lambda_{2}$, the correction $\Lambda_{3}$ appears to be highly suppressed with respect to a typical first order noncommutative contribution. This shows that our previous assumption on which the analytic result is based and that ignored $\Lambda$ corrections was not completely justifiable. Nevertheless, the mistake made by no means violates an accepted level of tolerance.

Hence, taking into account the WKB corrections $\Lambda_{2}$ and $\Lambda_{3}$, we obtain the graphs shown in Fig. 3, representing a behavior of the real and imaginary part of the fundamental frequency versus the charge of the scalar probe. The corresponding frequency splitting between the lines characterized with $m=100,0,-100$ is shown in Fig. 4. As already indicated, the $\mathrm{BH}$ parameters are $M=1, Q=0.5$, $l=100, m=-100,0,100$ and the charge $q$ of the scalar probe is in the range $q \in(1800,2200)$. In Fig. 4 we again

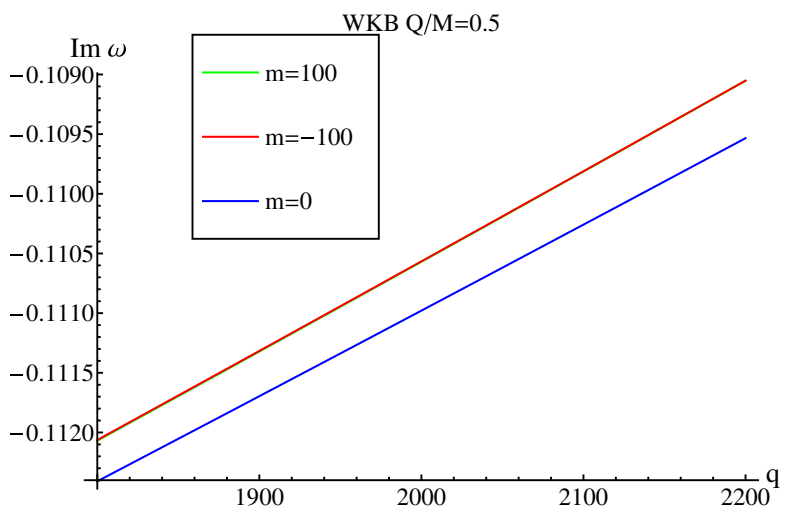

FIG. 3. Dependence of $\operatorname{Re} \omega$ (left) and $\operatorname{Im} \omega$ (right) on the scalar field charge $q$. 

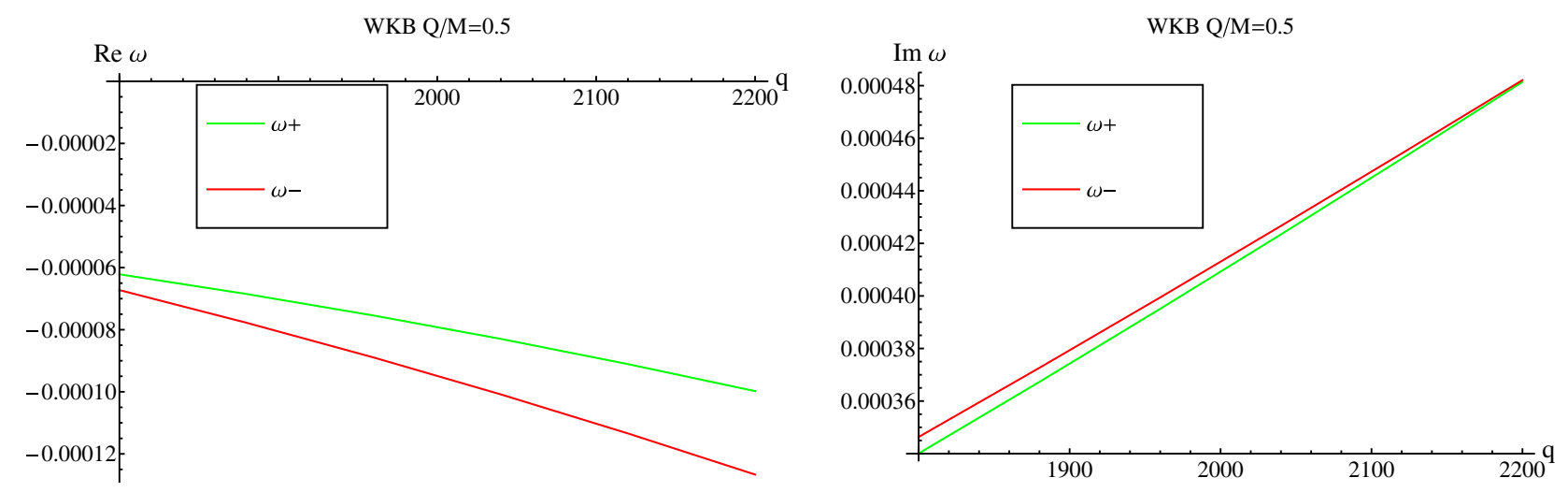

FIG. 4. Dependence of $\operatorname{Re} \omega$ (left) and $\operatorname{Im} \omega$ (right) splitting on the scalar field charge $q$.

note a splitting of the frequency lines for different $m$. However, this splitting is no more symmetric under the exchange $m \rightarrow-m$. It is broken by the higher WKB corrections.

As the potential $V$, after fixing the system parameters, depends on two variables, $r$ and $\omega$, a bit of explanation is needed to describe a set of steps undertaken in order to get the behavior of the fundamental frequency versus charge $q$. First, we have to solve the extremality condition $V^{\prime}=0$. The solution is going to give several outcomes that are all functions of the frequency $\omega$. To get a meaning out of that, we first insert into these solutions a classical value of the frequency $\omega=\frac{q Q}{r_{+}}$, which gives several values for $r_{0}$, some of them being complex numbers. Among all these values we take the one that is closest to and greater than the outer horizon $r_{+}$. This will obviously yield an extremal point $r_{0}$ that is only approximate. As the next step, we plug $r_{0}$ obtained in this way into the quantization condition (3.9) and find the corresponding root in the vicinity of the classical value $\frac{q Q}{r_{+}}$. This is going to give an improved value of the fundamental frequency. Subsequently, with this obtained frequency we turn back to the extremality condition and solve it again with that improved value for the fundamental frequency to get an improved solution for the extremal point $r_{0}$. Then we repeat this process iteratively until both quantities, the extremal point $r_{0}$ and the fundamental frequency that we search for, reach a satisfactory precision, i.e., until they both saturate at the values whose level of approximation is sufficiently high. After each round of iteration the values of $r_{0}$ and $\omega$ shift closer to their actual values. In essence, the actual (true) results for $r_{0}$ and $\omega$ come out as the values at which $r_{0}$ and $\omega$ saturate after a sufficient number of iterations has been carried out.

\section{CONTINUED FRACTION METHOD}

So far we used two different methods to calculate the QNM spectrum of NC scalar perturbation of the RN geometry. Both methods have their limitations. The WKB method works well for high values of the orbital momentum $l$. The analytic treatment in [18] was possible only in the near-extremal limit of the RN geometry.

In this section we implement the continued fraction method $[30,31]$ to determine the QNM spectrum of a massive charged scalar field around the RN black hole in the presence of the noncommutative deformation of spacetime. This method is less restrictive than the previous two, and we expect to find results for a wider range of parameters. We mention that the analysis of the undeformed (commutative) (un)charged scalar and Dirac QNM spectrum in the RN background by the continued fraction method can be found in $[28,32,33]$. To our knowledge, this is the first time that the continued fraction method is applied also to the NC deformations of the commutative case.

We start by looking for the asymptotic form of the QNM spectrum in the spatial infinity $r \rightarrow \infty$ and near the horizon $r \rightarrow r_{+}$. The asymptotic form can be obtained by analytically solving Eq. (2.17) in the asymptotic limits of the spatial infinity $r \rightarrow \infty$ and the event horizon $r \rightarrow r_{+}$and imposing the QNM boundary condition of purely incoming waves on the horizon and purely outgoing waves in the infinity.

In the $r \rightarrow \infty$ limit Eq. (2.17) reduces to

$$
\begin{aligned}
& \frac{\mathrm{d}^{2} \psi}{\mathrm{d} y^{2}}+\left[\omega^{2}-\mu^{2}-2 \frac{\omega q Q-\mu^{2} M}{r}+2 \operatorname{iam} \frac{q Q}{r}\left(\omega^{2}-\mu^{2}\right)\right] \psi \\
& \quad=0 .
\end{aligned}
$$

The solution to Eq. (4.1) is given by

$$
R=\frac{\psi}{r} \sim e^{ \pm i \Omega y} y^{-1-i \frac{\omega q Q-\mu^{2} M}{\Omega}-a m q Q \Omega} .
$$

Note that, in the limit $r \rightarrow \infty$, the parameter $\Omega$ is fixed by the leading order solution to $\Omega^{2}=\omega^{2}-\mu^{2}$. Analogously, in the near horizon limit $r \rightarrow r_{+}$, the associated equation of motion (3.1) reduces to

$$
\frac{\mathrm{d}^{2} \psi}{\mathrm{d} y^{2}}+\left[\omega-\frac{q Q}{r_{+}}\right]^{2}\left[1+2 \operatorname{iam} \frac{q Q}{r_{+}}\right] \psi=0,
$$

with the solution 


$$
\psi \sim e^{ \pm i\left(\omega-\frac{q Q}{r_{+}}\right)\left(1+i a m \frac{q Q}{r_{+}}\right) y} .
$$

Solutions (4.2) and (4.4) are perturbative in the NC parameter $a$ and are valid up to first order in $a$. The QNM boundary conditions, purely outgoing in the infinity and purely incoming at the horizon, select signs in (4.2) and (4.4). Finally, the asymptotic form of the quasinormal modes is given by

$$
R(r) \rightarrow\left\{\begin{array}{ll}
Z_{\text {out }} e^{i \Omega y} y^{-1-i \frac{\omega q Q-\mu^{2} M}{\Omega}-a m q Q \Omega}, & \text { for } r \rightarrow \infty(y \rightarrow \infty) \\
Z_{\text {in }} e^{-i\left(\omega-\frac{q Q}{r_{+}}\right)\left(1+i a m \frac{q Q}{r_{+}}\right) y}, & \text { for } r \rightarrow r_{+}(y \rightarrow-\infty)
\end{array} .\right.
$$

Here $Z_{\text {out }}$ and $Z_{\text {in }}$ are the amplitudes of the outgoing and ingoing waves, respectively, and they do not depend on $r$ (or $y$ ). In the special case of a massless scalar field $\mu=0$ and a vanishing spacetime deformation $a=0$, these asymptotic solutions reduce to the asymptotic solutions of [32].

Equation (2.17) has an irregular singularity at $r=+\infty$ and three regular singularities at $r=0, r=r_{-}$, and $r=r_{+}$. To implement Leaver's method we expand the solution in terms of power series around $r=r_{+}$. Then the radial part of the scalar field looks as

$$
R(r)=e^{i \Omega r}\left(r-r_{-}\right)^{\epsilon} \sum_{n=0}^{\infty} a_{n}\left(\frac{r-r_{+}}{r-r_{-}}\right)^{n+\delta} .
$$

The parameters $\delta$ and $\epsilon$ are determined by demanding that the solution (4.6) satisfies the boundary conditions (4.5) at the horizon and in the infinity. From the general form (4.6) of the solution, it is clear that as $r \rightarrow \infty$, the dominant behavior is determined by the term $r^{\epsilon} e^{i \Omega r}$. Likewise, as $r \rightarrow r_{+}$, the dominant behavior of (4.6) is given by the term $\left(r-r_{+}\right)^{\delta}$. On the other side, we insert the expression (3.7) for the tortoise coordinate into (4.2) and (4.4) and then compare the resulting expressions with the formerly deduced asymptotic of (4.6). From this comparison the parameters $\epsilon$ and $\delta$ follow immediately, and they are given by

$$
\begin{aligned}
& \delta=-i \frac{r_{+}^{2}}{r_{+}-r_{-}}\left(\omega-\frac{q Q}{r_{+}}\right), \\
& \epsilon=-1-i q Q \frac{\omega}{\Omega}+i \frac{r_{+}+r_{-}}{2 \Omega}\left(\Omega^{2}+\omega^{2}\right) .
\end{aligned}
$$

It is worth noting that these parameters are the same as the corresponding ones in Ref. [33] and thus are not affected by the noncommutative spacetime deformation.

\section{A. Recurrence relations}

Now we insert the power series solution (4.6) together with (4.7) into Eq. (2.17). In this way we obtain the recurrence relations for the coefficients $a_{n}$. The calculation leading to the recurrence relations is long and it is presented in Appendix A. The result is the six-term recurrence relation

$$
\begin{aligned}
A_{n} a_{n+1}+B_{n} a_{n}+C_{n} a_{n-1}+D_{n} a_{n-2}+E_{n} a_{n-3}+F_{n} a_{n-4}=0, & n \geq 4, \\
A_{3} a_{4}+B_{3} a_{3}+C_{3} a_{2}+D_{3} a_{1}+E_{3} a_{0}=0, & n=3, \\
A_{2} a_{3}+B_{2} a_{2}+C_{2} a_{1}+D_{2} a_{0}=0, & n=2, \\
A_{1} a_{2}+B_{1} a_{1}+C_{1} a_{0}=0, & n=1, \\
A_{0} a_{1}+B_{0} a_{0}=0, & n=0 .
\end{aligned}
$$

The coefficients $A_{n}, B_{n}, C_{n}, D_{n}, E_{n}$, and $F_{n}$ are given by

$$
\begin{aligned}
A_{n}= & r_{+}^{3} \alpha_{n}, \quad F_{n}=-r_{-}^{3} \gamma_{n-3}, \\
B_{n}= & r_{+}^{3} \beta_{n}-3 r_{+}^{2} r_{-} \alpha_{n-1}-i a m q Q\left(r_{+}-r_{-}\right) r_{+}(n+\delta)-\frac{1}{2} i a m q Q\left(r_{+}+r_{-}\right) r_{+}+i a m q Q r_{+} r_{-}, \\
C_{n}= & r_{+}^{3} \gamma_{n}+3 r_{+} r_{-}^{2} \alpha_{n-2}-3 r_{+}^{2} r_{-} \beta_{n-1}+i a m q Q\left(r_{+}-r_{-}\right)\left(2 r_{+}+r_{-}\right)(n+\delta-1)-i a m q Q\left(r_{+}-r_{-}\right) r_{+} \epsilon \\
& +\frac{1}{2} i a m q Q\left(r_{+}+r_{-}\right)\left(2 r_{+}+r_{-}\right)-3 i a m q Q r_{+} r_{-}+a m q Q \Omega\left(r_{+}-r_{-}\right)^{2} r_{+}, \\
D_{n}= & -r_{-}^{3} \alpha_{n-3}+3 r_{+} r_{-}^{2} \beta_{n-2}-3 r_{+}^{2} r_{-} \gamma_{n-1}+i a m q Q\left(r_{+}^{2}-r_{-}^{2}\right) \epsilon+3 i a m q Q r_{+} r_{-}-a m q Q \Omega\left(r_{+}-r_{-}\right)^{2} r_{-} \\
& -i a m q Q\left(r_{+}-r_{-}\right)\left(r_{+}+2 r_{-}\right)(n+\delta-2)-\frac{1}{2} i a m q Q\left(r_{+}+r_{-}\right)\left(r_{+}+2 r_{-}\right), \\
E_{n}= & 3 r_{+} r_{-}^{2} \gamma_{n-2}-r_{-}^{3} \beta_{n-3}+i a m q Q\left(r_{+}-r_{-}\right) r_{-}(n+\delta-3)-i a m q Q\left(r_{+}-r_{-}\right) r_{-} \epsilon+\frac{1}{2} i a m q Q\left(r_{+}+r_{-}\right) r_{-} \\
& +i a m q Q r_{+} r_{-} .
\end{aligned}
$$


The coefficients $\alpha_{n}, \beta_{n}, \gamma_{n}$ are

$$
\begin{aligned}
\alpha_{n}= & (n+1)\left[n+1-2 i \frac{r_{+}}{r_{+}-r_{-}}\left(\omega r_{+}-q Q\right)\right], \\
\beta_{n}= & \epsilon+(n+\delta)(2 \epsilon-2 n-2 \delta)+2 i \Omega(n+\delta)\left(r_{+}-r_{-}\right)-l(l+1)-\mu^{2} r_{-}^{2} \\
& +\frac{2 \omega r_{-}^{2}}{r_{+}-r_{-}}\left(\omega r_{+}-q Q\right)-\frac{2 r_{-}^{2}}{\left(r_{+}-r_{-}\right)^{2}}\left(\omega r_{+}-q Q\right)^{2}+4 \omega r_{-}\left(\omega r_{+}-q Q\right) \\
& -\frac{2 r_{-}}{r_{+}-r_{-}}\left(\omega r_{+}-q Q\right)^{2}+\left(r_{+}-r_{-}\right)\left[i \Omega+2 \omega\left(\omega r_{+}-q Q\right)-\mu^{2}\left(r_{+}+r_{-}\right)\right], \\
\gamma_{n}= & \epsilon^{2}+(n+\delta-1)(n+\delta-1-2 \epsilon)+\left(\omega r_{-}-\frac{r_{-}}{r_{+}-r_{-}}\left(\omega r_{+}-q Q\right)\right)^{2} .
\end{aligned}
$$

Let us clarify these relations. The first relation in (4.8), for $n \geq 4$, is a general six-term recurrence relation. The remaining four relations are the indicial equations relating the lowest order coefficients $a_{n}$ in the general expansion (4.6). They may be thought of as boundary conditions for the first relation in (4.8). The presence of $\mathrm{NC}$ deformation, through the terms linear in the NC parameter $a$, induces the six-term recurrence relation. In the commutative case and for the nonextremal RN background the three-term recurrence relation is obtained [32,33].

To compare the commutative limit of our result (4.8) with the results in the commutative case, we have to go back to Eq. (A8). There we impose the commutative limit $a \rightarrow 0$ and divide by $\left(r_{+}-z r_{-}\right)^{3}$ the remaining terms. The obtained equation then results in the three-term recurrence relation of $[32,33]$,

$$
\begin{aligned}
\alpha_{n} a_{n+1}+\beta_{n} a_{n}+\gamma_{n} a_{n-1} & =0, \\
\alpha_{0} a_{1}+\beta_{0} a_{0} & =0,
\end{aligned}
$$

with the coefficients $\alpha_{n}, \beta_{n}$, and $\gamma_{n}$ given in (4.10). Note that the noncommutativity does not influence the parameters $\alpha_{n}, \beta_{n}$, and $\gamma_{n}$.

Having the recurrence relations that involve more than three expansion coefficients $a_{n}$, as we do have here, we cannot straightforwardly apply the usual method for solving the recurrence relations [34]. Instead, we should first use the Gauss elimination method to gradually reduce the initial recurrence relation from the six-term recurrence relation to a three-term recurrence relation. In our case the Gauss elimination method needs to be applied 3 times in a row. The details of this calculation are presented in Appendix B. The final result is the three-term recurrence relation

$$
\begin{aligned}
A_{n}^{(3)} a_{n+1}+B_{n}^{(3)} a_{n}+C_{n}^{(3)} a_{n-1} & =0, \\
A_{0}^{(3)} a_{1}+B_{0}^{(3)} a_{0} & =0 .
\end{aligned}
$$

The coefficients $A_{n}^{(3)}, B_{n}^{(3)}, C_{n}^{(3)}$ are functions of the original coefficients $A_{n}, \ldots, F_{n}$. The explicit dependence is calculated in Appendix B; see (B9) and (B10).

To solve the three-term recurrence relation (4.12), we follow [30]. Since $r_{+}$is a regular singular point, the general expansion (4.6) converges for $r_{+} \leq r<\infty$. Demanding convergence at $r=\infty$ implies that the sum $\sum_{n} a_{n}$ also converges. Therefore, defining the quantity $R_{n}$ by $R_{n}=$ $-\frac{a_{n+1}}{a_{n}}$, we see that the infinite series (4.6) will converge if $R_{n}$ decreases sufficiently fast with the increase of $n$. Moreover, from the first relation in (4.12) we see that the coefficients $a_{n}$ must satisfy the recurrence relation

$$
\begin{aligned}
& R_{n-1}=\frac{C_{n}^{(3)}}{B_{n}^{(3)}-A_{n}^{(3)} R_{n}}, \quad \text { that is, } \\
& \frac{a_{n}}{a_{n-1}}=\frac{-C_{n}^{(3)}}{B_{n}^{(3)}-\frac{A_{n}^{(3)} C_{n+1}^{(3)}}{B_{n+1}^{(3)}-\frac{A_{n+1}^{(3)} C_{n+2}^{(3)}}{B_{n+2}^{(3)}}}} .
\end{aligned}
$$

This relation, in combination with the second relation in (4.12) leads to the infinite continued fraction equation

$$
0=B_{0}^{(3)}-\frac{A_{0}^{(3)} C_{1}^{(3)}}{B_{1}^{(3)}-\frac{A_{1}^{(3)} C_{2}^{(3)}}{B_{2}^{(3)}-\frac{A_{2}^{(3)} C_{3}^{(3)}}{B_{3}^{(3)}-\frac{A_{n}^{(3)} C_{n+1}^{(3)}}{B_{n+1}^{(3)} \cdots}}}} .
$$

The quantity $R_{n}$ may be interpreted as being the remaining part of the infinite continued fraction. It is supposed to decrease relatively fast as $n$ grows. Hence, the convergence of the series (4.6) is ensured if the coefficients $a_{n}$ satisfy Eq. (4.14) and $R_{n}$ decreases with increasing $n$. The solution to this infinite continued fraction equation gives the QNM frequencies. The continued fraction relation (4.14) can be inverted any number of times. Numerically, the $n$th QNM frequency is defined to be the most stable root of the $n$th 

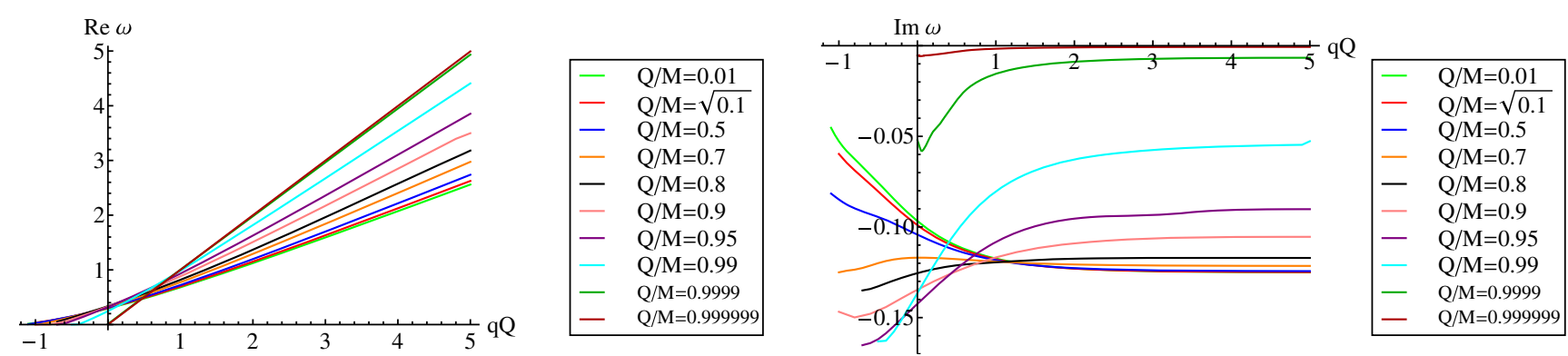

FIG. 5. Dependence of $\operatorname{Re} \omega$ (left) and $\operatorname{Im} \omega$ (right) on the charge $q Q$ of the scalar field with the mass $\mu=0.05$ and the orbital momentum $l=1$. Different extremalities are shown in different colors.

inversion of the continued fraction relation (4.14),

$$
\begin{aligned}
B_{n}^{(3)}-\frac{A_{n-1}^{(3)} C_{n}^{(3)}}{B_{n-1}^{(3)}-\frac{A_{n-2}^{(3)} C_{n-1}^{(3)}}{B_{n-2}^{(3)}-\cdots \frac{A_{0}^{(3)} C_{1}^{(3)}}{B_{0}^{(3)}}}} & =\frac{A_{n}^{(3)} C_{n+1}^{(3)}}{B_{n+1}^{(3)}-\frac{A_{n+1}^{(3)} C_{n+2}^{(3)}}{B_{n+2}^{(3)}-\cdots}}, \\
n & =1,2,3 \ldots
\end{aligned}
$$

The fundamental mode is obtained as the most stable root of Eq. (4.14).

\section{B. Numerical results}

Now we use the root finding algorithm (Wolfram Mathematica) to determine the QNM spectrum from Eqs. (4.14) and (4.15). The NC parameter $a$ is fixed to $a=0.01$. We present our results graphically.

The dependence of the fundamental QNM frequency $\omega=\operatorname{Re} \omega+i \operatorname{Im} \omega$ on the charge ${ }^{2}$ of the scalar field $q Q$ is shown in Fig. 5. The remaining parameters are fixed as follows: $\mu=0.05, l=1$, and $M=1$ in accordance with [32,33]. The extremality of the RN black hole is controlled by the value of $\frac{Q}{M}$. We present results for ten different cases, with $\frac{Q}{M}$ varying from 0.01 to 0.999999 . One can see immediately from Fig. 5 that, as the electromagnetic interaction increases ( $q Q$ increases), the imaginary part of the quasinormal frequency approaches a constant value, while the real part seems to grow linearly. In addition, as $q Q$ decreases, the real part of the fundamental frequency Re $\omega$ approaches zero at some critical value of the electromagnetic coupling. This behavior is similar to the behavior in the commutative case [32]. As this critical value is approached, the continued fraction method seems to converge slower.

The imaginary part of the fundamental frequency $\operatorname{Im} \omega$ changes its behavior as the extremal limit is approached, at some point between $\frac{Q}{M}=0.8$ and $\frac{Q}{M}=0.9$. As the extremal limit $\frac{Q}{M}=1$ is approached, the imaginary part of the

\footnotetext{
${ }^{2}$ The charge of the $\mathrm{RN}$ black hole $Q$ is fixed.
}

fundamental frequency becomes smaller, while the real part approaches $\frac{q Q}{r_{+}} \approx q$.

The existence of the modes with an arbitrarily small imaginary part in the near-extremal limit could be an artifact of the complicated continued fraction equation, as suggested in [32]. Namely, Leaver's original method fails to converge for extremal black holes. More precisely, when $\frac{Q}{M} \rightarrow 1$, the regular singularities at $r=r_{+}$and $r=r_{-}$ merge at $r=M$, becoming an irregular singularity. Therefore, it is not expected that a power series expansion around $r=r_{+}$will have a nonzero radius of convergence. Onozawa et al. [35] have proposed a modification of Leaver's method to deal with this type of equations and have successfully applied it to uncharged fields around an extremal RN black hole. The obtained results are in very good agreement with the ones obtained for nearly extremal black holes using Leaver's original method. In our previous work [18], we treated Eq. (2.17) analytically in the near extremal limit and we verified the existence of the modes with arbitrary small imaginary parts. In fact, the QNMs obtained in [18] in the near extremal limit are in excellent agreement with the ones obtained through the continued fraction method in the limit $\frac{Q}{M} \rightarrow 1$.

In Fig. 6 we show the dependence of the fundamental frequency $\operatorname{Re} \omega$ and $\operatorname{Im} \omega$ on the mass $\mu$ of the scalar field $\phi$. The remaining parameters are fixed to $q Q=1, l=1$, and $M=1$. Different extremalities are shown in different colors. The value of $\frac{Q}{M}$ varies from 0.01 up to 0.9999 . The existence of quasiresonances, special values of the mass when I $m \omega=0$, is clearly visible for all extremalities presented. The existence of quasiresonances is in agreement with the results obtained in the near-extremal approximation in [18].

Finally, let us comment on the effects of noncommutative deformation in our model. It is clear from Eqs. (4.8) and (4.9) that the QNM frequencies depend on the value of $a m$, where $a$ controls the NC deformation and $m=$ $-l,-l+1, \ldots, l$ is the projection of the orbital momentum $l$. Therefore, we expect to find a frequency splitting for different values of $m$. To make this observation more explicit, in Figs. 7 and 8 we show the frequency splitting 

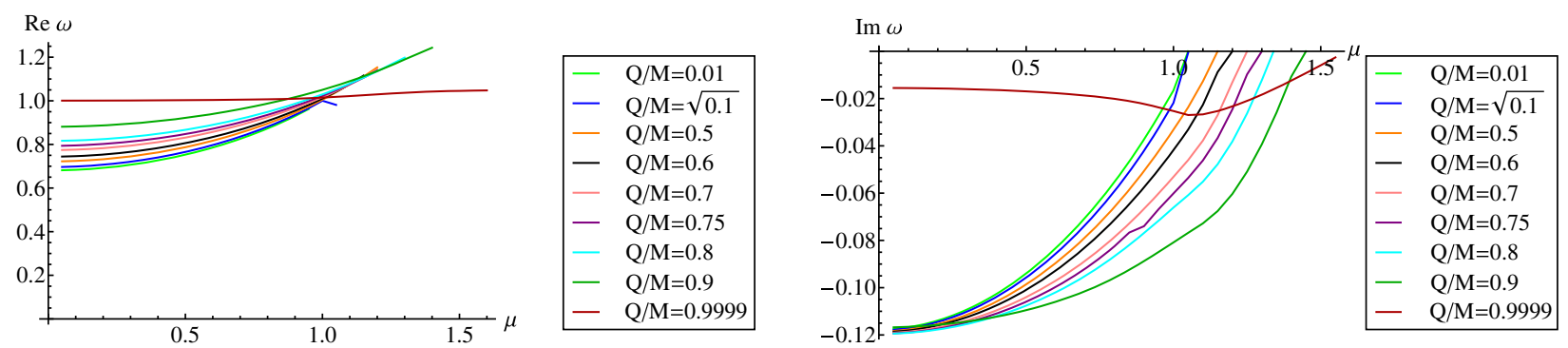

FIG. 6. Dependence of $\operatorname{Re} \omega$ (left) and Im $\omega$ (right) on the mass $\mu$ of the scalar field with the charge $q Q=1$ and the orbital momentum $l=1$.
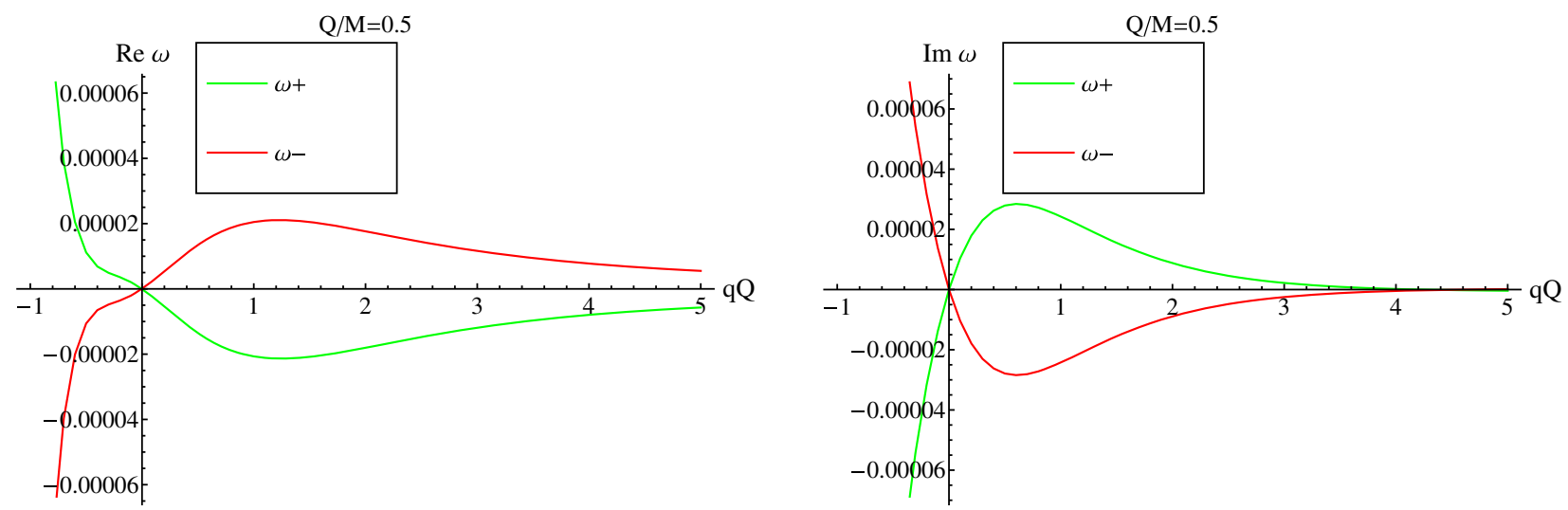

FIG. 7. Dependence of $\operatorname{Re} \omega^{ \pm}$(left) and $\operatorname{Im} \omega^{ \pm}$(right) on the charge $q Q$ of the scalar field with the mass $\mu=0.05$, orbital momentum $l=1$, and extremality $\frac{Q}{M}=0.5$.

as a function of the scalar field charge $q$ (Fig. 7) and of the scalar field mass $\mu$ (Fig. 8). The splitting can be graphically presented for an arbitrary value of $\frac{Q}{M}$, taking into account comments on the near extremal limit from the previous paragraph. In the example shown in Figs. 7 and 8 we fixed the extremality to $\frac{Q}{M}=0.5$; that is, we show splitting in the nonextremal case. The splitting is defined as $\omega^{ \pm}=$ $\omega(m= \pm 1)-\omega(m=0)$.

The relative frequency splitting can be estimated from Figs. 5 and 7 and Figs. 6 and 8. For example, in the case of fixed mass $\mu=0.05$ and $\frac{Q}{M}=0.5$ we find $\delta_{\operatorname{Re}} \sim \frac{\operatorname{Re} \omega^{+}}{\operatorname{Re} \omega} \sim$ $10^{-4}$ and $\delta_{\operatorname{Im}} \sim \frac{\operatorname{Im} \omega^{+}}{\operatorname{Im} \omega} \sim 10^{-4}$. One can check that in the case of fixed charge $q Q=1$, the splitting is approximately of the same order.

It is important to stress that in the limit $a \rightarrow 0$ (and $\mu \rightarrow 0$ ) our results reduce to the results presented in $[28,32,33]$, meaning that we have been able to reproduce in full detail the same graph profiles as in these references. In particular, for the uncharged massive scalar field in the absence of noncommutativity and for $\frac{Q}{M}=\sqrt{0.1} \approx 0.316$

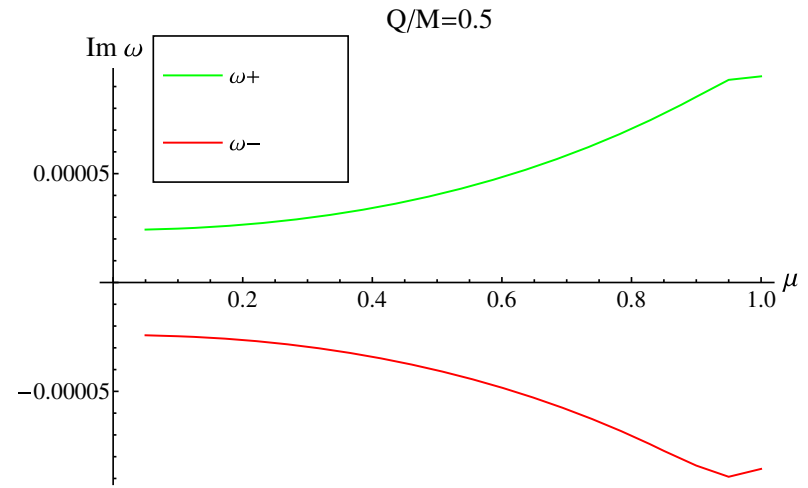

FIG. 8. Dependence of $\operatorname{Re} \omega^{ \pm}$(left) and $\operatorname{Im} \omega^{ \pm}$(right) on the mass $\mu$ of the scalar field with the charge $q Q=1$, orbital momentum $l=1$, and extremality $\frac{Q}{M}=0.5$. 

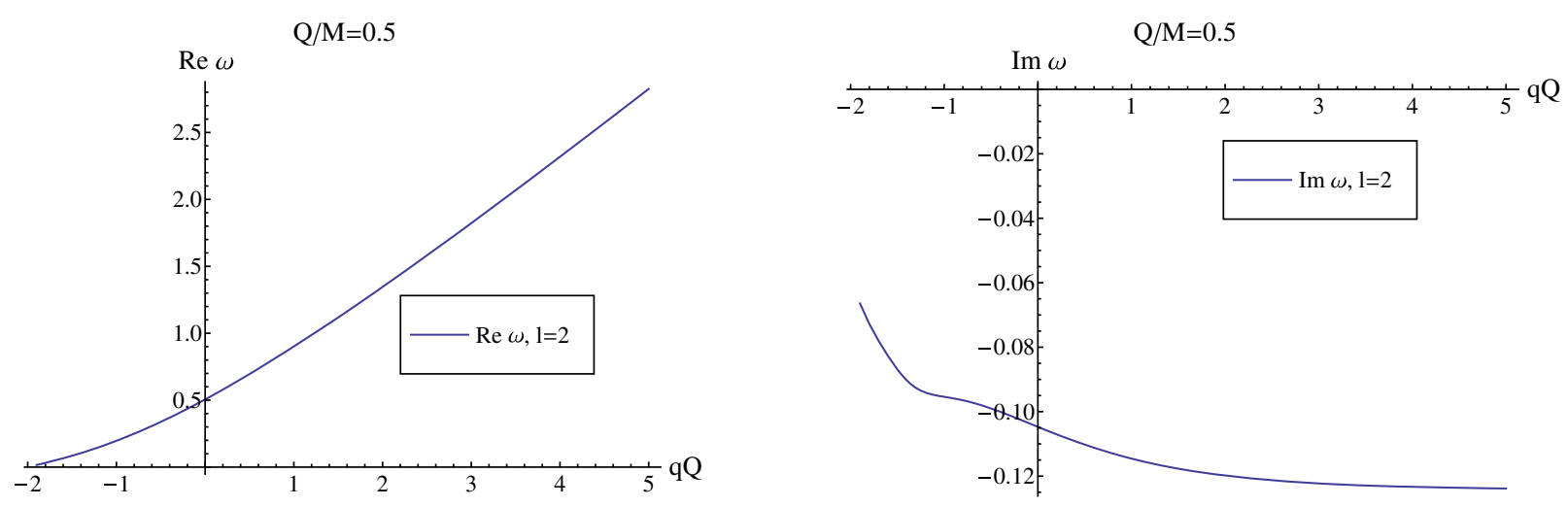

FIG. 9. Dependence of $\operatorname{Re} \omega$ (left) and $\operatorname{Im} \omega$ (right) on the charge $q Q$ of the scalar field with the mass $\mu=0.05$, the orbital momentum $l=2$, and the extremality $\frac{Q}{M}=0.5$.

we checked that the curves showing the dependence of $\operatorname{Re} \omega$ and $\operatorname{Im} \omega$ on the mass $\mu$ are in perfect agreement with the corresponding curves in Ref. [33] [see the graphs in Figs. 2(c) and 2(d), respectively], specified for the case of the scalar charge $s=0.1$. In Figs. 5 and 6 the dark blue lines correspond to this particular extremality, $\frac{Q}{M}=0.316$. However, in this case, unlike in [33] the scalar field is charged, and in addition there are effects of noncommutativity.

Another example that may be interesting to confront with our results is the fundamental mode of the massless scalar field in a nonrotational Kerr-Newman background that was studied in Ref. [28] (see Fig. 3 in [28] for the case $\left.r_{-}=0.95 r_{+}\right)$. This case roughly corresponds to the extremality ratio of $Q / M=0.9999$, which is represented on our Fig. 5 by the dark green line. A close inspection of two graph profiles shows a high level of agreement. In particular, the imaginary parts in both cases saturate roughly at the same value and both have the minimum lying on the vertical axis. The small discrepancy between the two profiles may be attributed to the fact that the mass of the scalar probe in these two cases is not the same, as well as to a small effect caused by noncommutativity.

\section{DISCUSSION AND FINAL REMARKS}

In this paper we studied the QNM spectrum of the NC scalar perturbation in the nonextremal RN background in more depth. In our previous work [18] we did the analytic analysis limited to the near-extremal geometry. Here we overcome this limitation by using the WKB and the continued fraction methods. The WKB method is well-defined for large values of the orbital momentum $l$, and due to our additional approximations is limited to the parameter region $l+1 \ll q Q$. In this approximation we solved the WKB condition analytically and plotted our results in Figs. 1 and 2. If one makes the additional approximation $l \ll q Q \ll l^{2}$, the massless, commutative limit of our results (3.20) and (3.21) agrees with the results obtained in [26]. We have also studied the influence of the higher order WKB corrections on the QNM spectrum for large $l$ and found that the next to leading order term $\Lambda_{2}$ in the quantization condition (3.9) may have a non-negligible effect.

Looking for a less restrictive method that is also able to describe the lower lying angular momentum channels in the QNM spectrum, we then turned to the continued fraction method. To our knowledge, this is the first time the continued fraction method is applied to the noncommutative QNM spectrum problem. The NC deformation induces the six-term recurrence relation (4.8), and we use the Gauss elimination procedure to reduce it to the three-term recurrence relation (4.12). We used the root finding algorithm to solve the three-term recurrence relation and obtain the fundamental QNM frequency. The results we obtain are plotted in Figs. 5-8 and are in good qualitative agreement with our previous results in [18]. We discuss some of the properties of the QNM spectrum in Sec. IV 2. Here, for completeness, we also show the results for $l=2$. In Fig. 9 we plot the dependence of the fundamental frequency $\omega$ on the scalar field charge $q$, while in Fig. 10 we plot the corresponding frequency splitting defined as $\omega^{ \pm \pm}=\omega(m= \pm 2)-\omega(m=0)$.

It is, of course, important to connect our results with realistic astrophysical black holes. To do that, we first have to restore all fundamental constants $(\hbar, G, c)$ to our equations. Equation of motion (2.17) has the following form:

$$
\begin{gathered}
\hbar^{2} f R_{l m}^{\prime \prime}+\frac{2 \hbar^{2}}{r}\left(1-\frac{M G}{r c^{2}}\right) R_{l m}^{\prime}-\left(\frac{l(l+1) \hbar^{2}}{r^{2}}-\frac{1}{f}\left(\frac{\hbar \omega}{c}-\frac{q Q}{4 \pi \epsilon_{0} c r}\right)^{2}+\mu^{2} c^{2}\right) R_{l m} \\
-i m a \frac{q Q}{4 \pi \epsilon_{0} r^{3}} \hbar\left(\left(\frac{M G}{r c^{2}}-\frac{G Q^{2}}{4 \pi \epsilon_{0} c^{4} r^{2}}\right) R_{l m}+r f R_{l m}^{\prime}\right)=0 .
\end{gathered}
$$


The recurrence relations (4.9) are now replaced by

$$
\begin{aligned}
A_{n}= & r_{+}^{3} \alpha_{n}, \\
B_{n}= & r_{+}^{3} \beta_{n}-3 r_{+}^{2} r_{-} \alpha_{n-1}-\frac{i a m q Q}{4 \pi \epsilon_{0} \hbar}\left(r_{+}-r_{-}\right) r_{+}(n+\delta)-\frac{1}{2} \frac{i a m q Q}{4 \pi \epsilon_{0} \hbar}\left(r_{+}+r_{-}\right) r_{+}+\frac{i a m q Q}{4 \pi \epsilon_{0} \hbar} r_{+} r_{-}, \\
C_{n}= & r_{+}^{3} \gamma_{n}+3 r_{+} r_{-}^{2} \alpha_{n-2}-3 r_{+}^{2} r_{-} \beta_{n-1}+\frac{i a m q Q}{4 \pi \epsilon_{0} \hbar}\left(r_{+}-r_{-}\right)\left(2 r_{+}+r_{-}\right)(n+\delta-1)-\frac{i a m q Q}{4 \pi \epsilon_{0} \hbar}\left(r_{+}-r_{-}\right) r_{+} \epsilon \\
& +\frac{1}{2} \frac{i a m q Q}{4 \pi \epsilon_{0} \hbar}\left(r_{+}+r_{-}\right)\left(2 r_{+}+r_{-}\right)-3 \frac{i a m q Q}{4 \pi \epsilon_{0} \hbar} r_{+} r_{-}+\frac{a m q Q}{4 \pi \epsilon_{0} \hbar} \Omega\left(r_{+}-r_{-}\right)^{2} r_{+}, \\
D_{n}= & -r_{-}^{3} \alpha_{n-3}+3 r_{+} r_{-}^{2} \beta_{n-2}-3 r_{+}^{2} r_{-} \gamma_{n-1}+\frac{i a m q Q}{4 \pi \epsilon_{0} \hbar}\left(r_{+}^{2}-r_{-}^{2}\right) \epsilon+3 \frac{i a m q Q}{4 \pi \epsilon_{0} \hbar} r_{+} r_{-} \\
& -\frac{a m q Q}{4 \pi \epsilon_{0} \hbar} \Omega\left(r_{+}-r_{-}\right)^{2} r_{-}-\frac{i a m q Q}{4 \pi \epsilon_{0} \hbar}\left(r_{+}-r_{-}\right)\left(r_{+}+2 r_{-}\right)(n+\delta-2)-\frac{1}{2} i a m q Q\left(r_{+}+r_{-}\right)\left(r_{+}+2 r_{-}\right), \\
E_{n}= & 3 r_{+} r_{-}^{2} \gamma_{n-2}-r_{-}^{3} \beta_{n-3}+\frac{i a m q Q}{4 \pi \epsilon_{0} \hbar}\left(r_{+}-r_{-}\right) r_{-}(n+\delta-3)-\frac{i a m q Q}{4 \pi \epsilon_{0} \hbar}\left(r_{+}-r_{-}\right) r_{-} \epsilon \\
& +\frac{1}{2} \frac{i a m q Q}{4 \pi \epsilon_{0} \hbar}\left(r_{+}+r_{-}\right) r_{-}+\frac{i a m q Q}{4 \pi \epsilon_{0} \hbar} r_{+} r_{-}, \\
F_{n}= & -r_{-}^{3} \gamma_{n-3},
\end{aligned}
$$

with coefficients $\alpha_{n}, \beta_{n}, \gamma_{n}$

$$
\begin{aligned}
\alpha_{n}= & (n+1)\left[n+1-2 i \frac{r_{+}^{2}}{\left(r_{+}-r_{-}\right) c}\left(\omega-\frac{q Q}{4 \pi \epsilon_{0} \hbar r_{+}}\right)\right], \\
\beta_{n}= & \epsilon+(n+\delta)(2 \epsilon-2 n-2 \delta)+\frac{2 i}{c} \Omega(n+\delta)\left(r_{+}-r_{-}\right)-l(l+1)-\frac{\mu^{2} c^{2}}{\hbar^{2}} r_{-}^{2} \\
& +\frac{2 \omega r_{-}^{2}}{c\left(r_{+}-r_{-}\right)}\left(\omega r_{+}-\frac{q Q}{4 \pi \epsilon_{0} \hbar}\right)-\frac{2 r_{-}^{2}}{c^{2}\left(r_{+}-r_{-}\right)^{2}}\left(\omega r_{+}-\frac{q Q}{4 \pi \epsilon_{0} \hbar}\right)^{2}+\frac{4}{c} \omega r_{-}\left(\omega r_{+}-\frac{q Q}{4 \pi \epsilon_{0} \hbar}\right) \\
& -\frac{2 r_{-}}{c\left(r_{+}-r_{-}\right)}\left(\omega r_{+}-\frac{q Q}{4 \pi \epsilon_{0} \hbar}\right)^{2}+\frac{\left(r_{+}-r_{-}\right)}{c}\left[i \Omega+\frac{2}{c} \omega\left(\omega r_{+}-\frac{q Q}{4 \pi \epsilon_{0} \hbar}\right)-\frac{\mu^{2} c^{3}}{\hbar^{2}}\left(r_{+}+r_{-}\right)\right], \\
\gamma_{n}= & \epsilon^{2}+(n+\delta-1)(n+\delta-1-2 \epsilon)+\left(\frac{\omega r_{-}}{c}-\frac{r_{-}}{c\left(r_{+}-r_{-}\right)}\left(\omega r_{+}-\frac{q Q}{4 \pi \epsilon_{0} \hbar}\right)\right)^{2} .
\end{aligned}
$$

In addition, the parameters $\delta, \epsilon$, and $\Omega$ are given by

$$
\begin{aligned}
\delta & =-i \frac{r_{+}^{2}}{c\left(r_{+}-r_{-}\right)}\left(\omega-\frac{q Q}{4 \pi \epsilon_{0} \hbar r_{+}}\right), \\
\epsilon & =-1-i \frac{q Q}{4 \pi \epsilon_{0} c \hbar} \frac{\omega}{\Omega}+i \frac{r_{+}+r_{-}}{2 \Omega c}\left(\Omega^{2}+\omega^{2}\right), \\
\Omega^{2} & =\omega^{2}-\frac{\mu^{2} c^{4}}{\hbar^{2}},
\end{aligned}
$$

while the RN metric $g_{\mu \nu}$ can be read from

$$
\mathrm{d} s^{2}=\left(c^{2}-\frac{2 M G}{r}+\frac{Q^{2} G}{4 \pi \epsilon_{0} r^{2} c^{2}}\right) \mathrm{d} t^{2}-\frac{\mathrm{d} r^{2}}{1-\frac{2 M G}{r c^{2}}+\frac{Q^{2} G}{4 \pi \epsilon_{0} r^{2} c^{4}}}-r^{2}\left(\mathrm{~d} \theta^{2}+\sin ^{2} \theta \mathrm{d} \varphi\right) .
$$

Second, we need to estimate the scale of noncommutativity, controlled by parameter $a$. Let us assume that the NC scale is at the current range of LHC

$$
\Lambda_{\mathrm{NC}} \sim 10 \mathrm{TeV}
$$



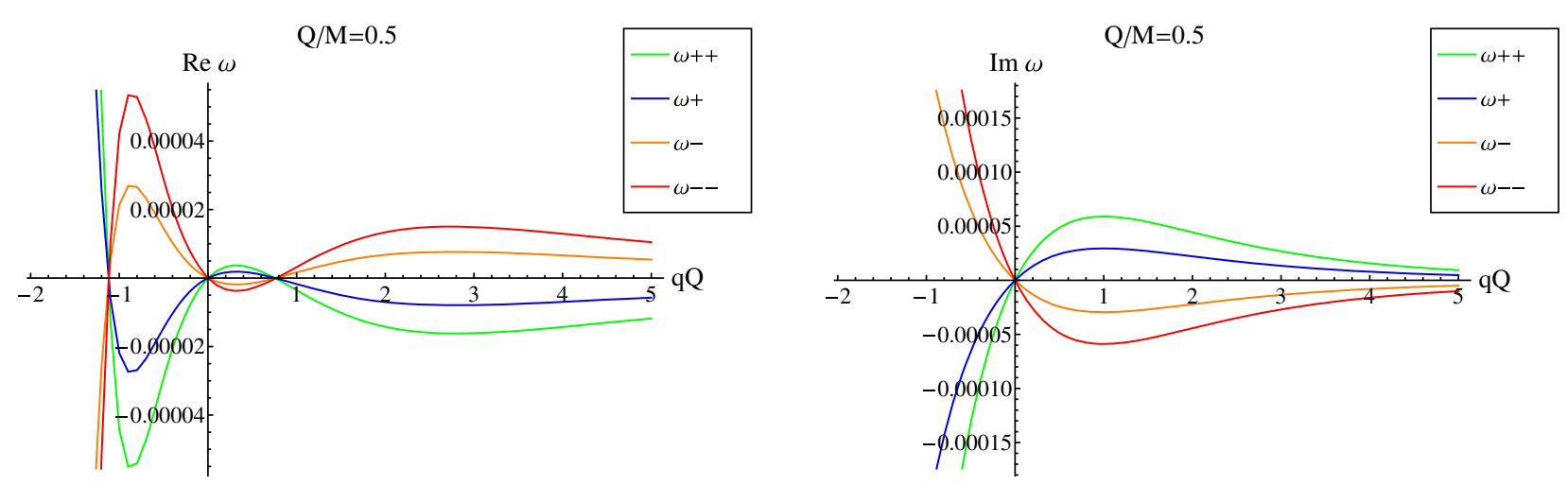

FIG. 10. Dependence of $\operatorname{Re} \omega^{ \pm}, \operatorname{Re} \omega^{ \pm \pm}$(left) and $\operatorname{Im} \omega^{ \pm}, \operatorname{Im} \omega^{ \pm \pm}$(right) on the charge $q Q$ of the scalar field with the mass $\mu=0.05$, orbital momentum $l=2$, and extremality $\frac{Q}{M}=0.5$.

In the case of Moyal-Weyl deformation, we have

$$
\left[x^{\mu}, x^{\nu}\right] \sim\left(\frac{\hbar c}{\Lambda_{\mathrm{NC}}}\right)^{2}
$$

In analogy, for the angular twist deformation, we can estimate

$$
[t, x] \sim\left(\frac{\hbar}{\Lambda_{\mathrm{NC}}}\right) x \sim a x
$$

that is,

$$
[t, \varphi] \sim\left(\frac{\hbar}{\Lambda_{\mathrm{NC}}}\right) \sim a
$$

With $\Lambda_{\mathrm{NC}} \sim 10 \mathrm{TeV}$ we find that the NC parameter $a$ can be estimated as

$$
a=6.25 \times 10^{-29} \mathrm{~s} .
$$

Finally, we need to fix mass $M$ of the RN black hole and its charge $Q$. We will consider two possibilities: supermassive BHs and primordial BHs.

A supermassive $\mathrm{BH}$ sitting in the center of a galaxy has a mass $M \sim 10^{6} M_{\odot}$, where $M_{\odot}=2 \times 10^{30} \mathrm{~kg}$ is the solar mass. Supermassive BHs are (almost) neutral. The maximal

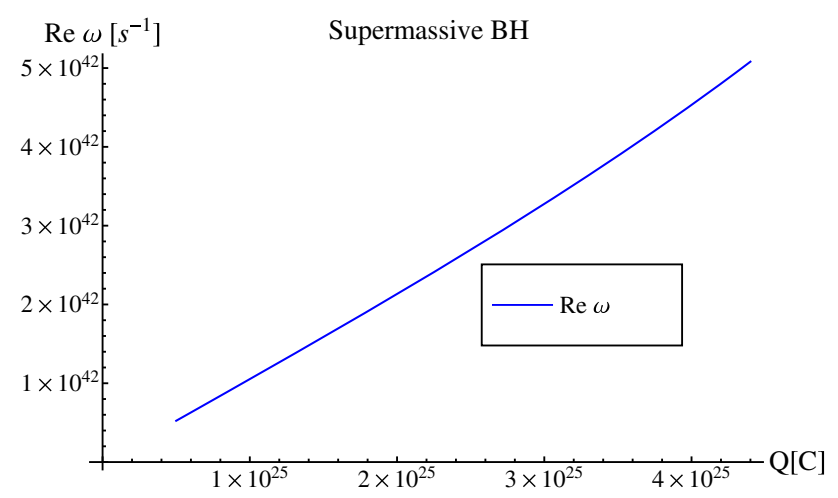

charge can be estimated to be $100 C$ per $M_{\odot}$; see, for example, [36].

In our example, we fix the $\mathrm{BH}$ mass to $M=10^{6} M_{\odot}$ and vary its charge so that $0 \leq Q \leq 4.4 \times 10^{6} \mathrm{C}$. For simplicity, we assume that the scalar field is massless, $\mu=0$, and it is charged with $q=e=1.6 \times 10^{-19} C$ and $l=1$. With all these parameters included, the continued fraction algorithm gives the dependences of $\operatorname{Re} \omega$ and $\operatorname{Im} \omega$ shown in Fig. 11. As in our previous examples in Secs. IV and V, the frequency splitting for $m=0, \pm 1$ exists. However, with this particular choice of parameters, the splitting is covered by the numerical error and is therefore unobservable.

Now we turn to the other example of primordial BHs. Primordial BHs were created in the early universe, shortly after the big bang. These BHs can have very small masses, but due to some limits as evaporation via Hawking radiation, their masses can be estimated to $M \sim 10^{12} \mathrm{~kg}$. In our example, we fix $M=8.8 \times 10^{12} \mathrm{~kg}$ and vary the $\mathrm{BH}$ charge within the interval $13 C \leq Q \leq 44 C$. As in the previous case, we take a massless scalar field with $q=$ $e$ and $l=1$. Results obtained by the continued fraction method are shown in Figs. 12 and 13. Figure 12 shows the dependences of $\operatorname{Re} \omega$ and $\operatorname{Im} \omega$ on the $\mathrm{BH}$ charge $Q$. Figure 13 shows the $\operatorname{Im} \omega$ frequency splitting for $m= \pm 1$. We can approximate the splitting with $\frac{\delta \omega}{\omega} \sim \frac{10^{14}}{10^{17}} \sim 10^{-3}$. The splitting of $\operatorname{Re} \omega$ is again unobservable.

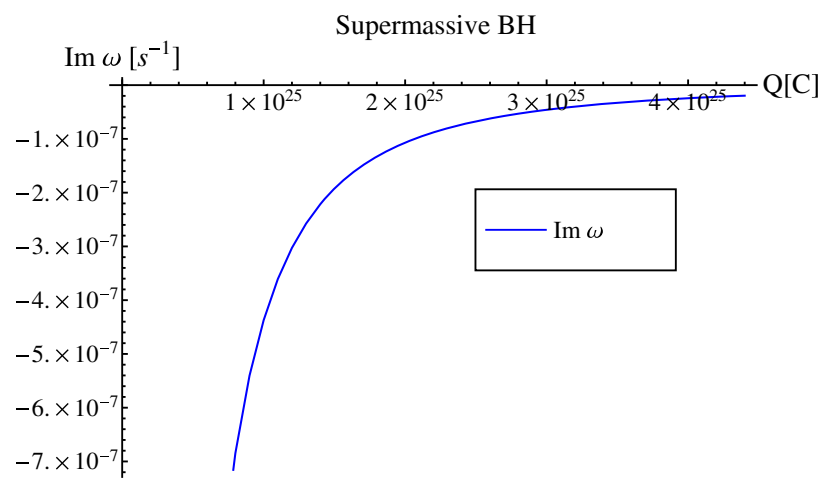

FIG. 11. Dependence of $\operatorname{Re} \omega$ (left) and $\operatorname{Im} \omega$ (right) on the $\mathrm{BH}$ charge $Q$. 

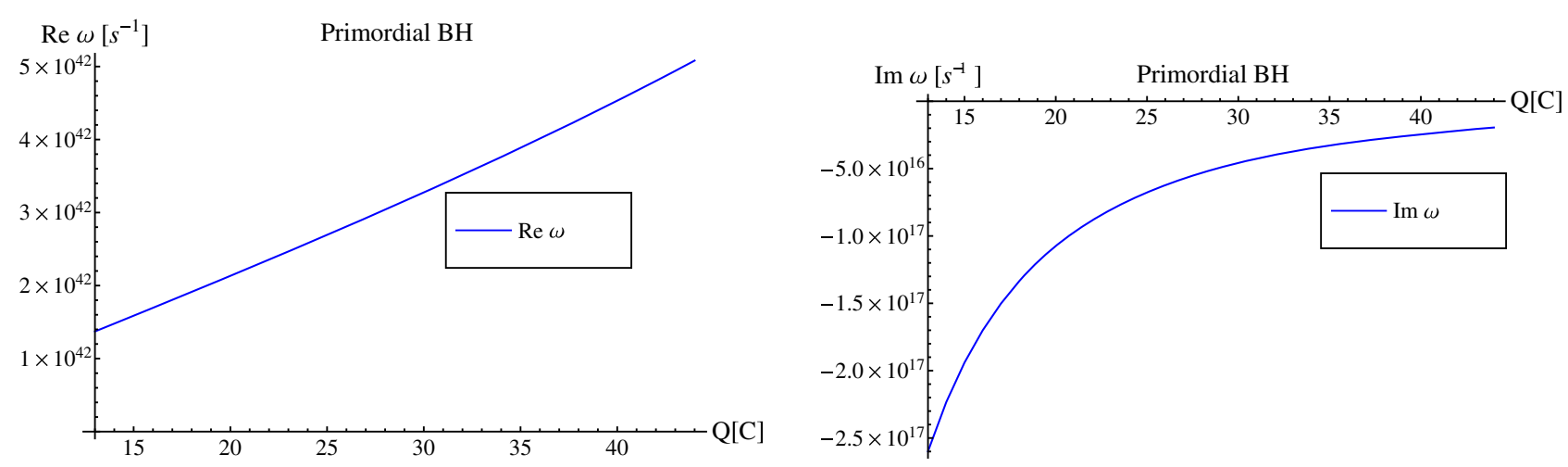

FIG. 12. Dependence of $\operatorname{Re} \omega$ (left) and $\operatorname{Im} \omega$ (right) on the BH charge $Q$.

As we mentioned before, this is the first time the continued fraction method is applied to a NC QNM spectrum problem. In our future work, we plan to investigate spinor and vector NC QNM spectrum. Of course, we are also interested in gravitational QNMs, since these can be experimentally detected as gravitational waves. However, to calculate the gravitational QNMs we have to go beyond the semiclassical analysis used in this paper. That is, we have to include effects of NC deformation on the gravitational field as well. There are different ways to approach this problem. One way is to construct a $\mathrm{NC}$ gravity action, or at least NC Einstein equations and look for explicit solutions describing NC black holes. It is our future plan to start from NC Einstein equations obtained in [37] and calculate gravitational QNMs.

On the other hand, there are phenomenological models of NC black holes that usually go by the name of "NC inspired black holes" [38]. These models are based on commutative (classical) Einstein equations with a source that is no longer pointlike (mass and charge), but replaced by a Gaussian energy density

$$
\rho_{M}=\frac{M}{(4 \pi \theta)^{3 / 2}} e^{-\frac{r^{2}}{4 \theta}}
$$

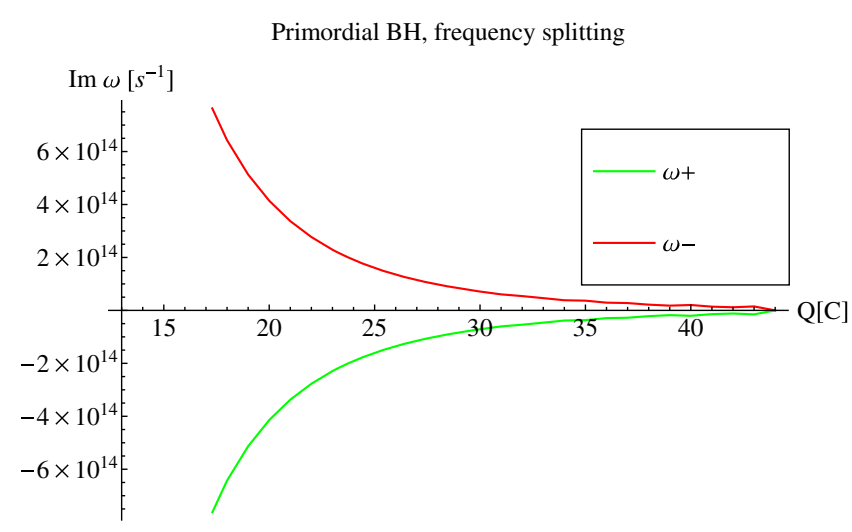

FIG. 13. Dependence of $\operatorname{Im} \omega^{ \pm}$on the BH charge $Q$. and similarly for the charge density. This choice of the energy-momentum tensor is well motivated by the coordinate coherent state approach $[39,40]$. However, the Einstein equation is classical, the classical covariant conservation of $T_{\mu \nu}$ is assumed, and in addition only the Schwarzschild-type of solutions, $g_{t t}=\frac{1}{g_{r r}}$, were considered. In addition to this, these models only consider Moyal-Weyl NC deformation. Therefore, we cannot directly include them in our current work, since our NC deformation is of the Lie-algebra type; see (2.5). It would be interesting to investigate possible extensions of $\mathrm{NC}$ inspired black holes to nonconstant $\mathrm{NC}$ deformations and connect that work with our model.

\section{ACKNOWLEDGMENTS}

We thank Mauricio Richartz, Tajron Jurić, and Svetislav Mijatović for fruitful discussion and useful comments. The work of M.D.C. and N.K. is supported by Project No. ON171031 of the Serbian Ministry of Education and Science. The work of A.S. is partially supported by the H2020 CSA Twinning Project No. 692194, RBI-T-WINNING, as well as by the project "Synergy to Success: RBI-T-WINNING and ESIF Associated in Strengthening the Excellence of the Institute of Theoretical Physics of the Rudjer Boskovic Institute (RBI-TWINN-SIN)." This work is partially supported by ICTP-SEENET-MTP Project No. NT-03 "CosmologyClassical and Quantum Challenges" in frame of the Southeastern European Network in Theoretical and Mathematical Physics and by the Action MP1405 QSPACE from the European Cooperation in Science and Technology (COST).

\section{APPENDIX A: FINDING THE SIX-TERM RECURRENCE RELATION}

In this appendix we derive the six-term recurrence relations (4.8) and find the explicit expressions for the coefficients $A_{n}, B_{n}, C_{n}, D_{n}, E_{n}, F_{n}$ that appear there. Starting with $R(r)=e^{i \Omega r} \psi(r)$, Eq. (2.17) reduces to 


$$
\begin{aligned}
(r- & \left.r_{+}\right)\left(r-r_{-}\right) \frac{\mathrm{d}^{2} \psi}{\mathrm{d} r^{2}}+\left[2(r-M)+2 i \Omega\left(r-r_{+}\right)\left(r-r_{-}\right)-i a m q Q \frac{\left(r-r_{+}\right)\left(r-r_{-}\right)}{r^{2}}\right] \frac{\mathrm{d} \psi}{\mathrm{d} r} \\
& +\left[2(r-M) i \Omega-\Omega^{2}\left(r-r_{+}\right)\left(r-r_{-}\right)-l(l+1)+\frac{r^{4}}{\left(r-r_{+}\right)\left(r-r_{-}\right)}\left(\omega-\frac{q Q}{r}\right)^{2}\right. \\
& \left.-\mu^{2} r^{2}-i a m q Q\left(\frac{M}{r^{2}}-\frac{Q^{2}}{r^{3}}\right)-i a m q Q i \Omega \frac{\left(r-r_{+}\right)\left(r-r_{-}\right)}{r^{2}}\right] \psi=0 .
\end{aligned}
$$

Introducing the new variable $z=\frac{r-r_{+}}{r-r_{-}}$, one has

$$
r=\frac{r_{+}-z r_{-}}{1-z}, \quad r-r_{-}=\frac{r_{+}-r_{-}}{1-z}, \quad r-r_{+}=\frac{z\left(r_{+}-r_{-}\right)}{1-z}
$$

Noting that (2.17) has an irregular singularity at $r=+\infty$ and three regular singularities at $r=0, r=r_{-}$, and $r=r_{+}$, we can expand the solution in terms of powers series around $r=r_{+}$,

$$
\psi(r)=\left(r-r_{-}\right)^{\epsilon} \sum_{n=0}^{\infty} a_{n}\left(\frac{r-r_{+}}{r-r_{-}}\right)^{n+\delta}=\frac{\left(r_{+}-r_{-}\right)^{\epsilon}}{(1-z)^{\epsilon}} \sum_{n=0}^{\infty} a_{n} z^{n+\delta}
$$

We have to keep in mind that the complete radial solution

$$
R(r)=e^{i \Omega r}\left(r-r_{-}\right)^{\epsilon} \sum_{n=0}^{\infty} a_{n}\left(\frac{r-r_{+}}{r-r_{-}}\right)^{n+\delta}
$$

must satisfy the boundary conditions (4.5) on the horizon and at far infinity. The change of coordinates from $r$ to $z$ is accompanied with the transformation of derivatives

$$
\frac{\mathrm{d} F}{\mathrm{~d} r}=\frac{(1-z)^{2}}{r_{+}-r_{-}} \frac{\mathrm{d} F}{\mathrm{~d} z}, \quad \frac{\mathrm{d}^{2} F}{\mathrm{~d} r^{2}}=\frac{(1-z)^{3}}{\left(r_{+}-r_{-}\right)^{2}}\left[(1-z) \frac{\mathrm{d}^{2} F}{\mathrm{~d} z^{2}}-2 \frac{\mathrm{d} F}{\mathrm{~d} z}\right]
$$

Inserting further the expansion (A3) into (A1), and using the decomposition $r=\frac{r_{+}-z r_{-}}{1-z}=r_{-}+\frac{r_{+}-r_{-}}{1-z}$, results in

$$
\begin{aligned}
z(1 & -z)^{2}\left(r_{+}-r_{-}\right)^{\epsilon}\left[\frac{\epsilon(\epsilon+1)}{(1-z)^{\epsilon+2}} \sum_{n=0}^{\infty} a_{n} z^{n+\delta}+\frac{2 \epsilon}{(1-z)^{\epsilon+1}} \sum_{n=0}^{\infty} a_{n}(n+\delta) z^{n+\delta-1}+\frac{1}{(1-z)^{\epsilon}} \sum_{n=0}^{\infty} a_{n}(n+\delta)(n+\delta-1) z^{n+\delta-2}\right] \\
+ & {\left[(1-z)^{2}+2 i \Omega z\left(r_{+}-r_{-}\right)-i a m q Q \frac{\left(r_{+}-r_{-}\right) z(1-z)^{2}}{\left(r_{+}-z r_{-}\right)^{2}}\right]\left(r_{+}-r_{-}\right)^{\epsilon} } \\
& \times\left[\frac{\epsilon}{(1-z)^{\epsilon+1}} \sum_{n=0}^{\infty} a_{n} z^{n+\delta}+\frac{1}{(1-z)^{\epsilon}} \sum_{n=0}^{\infty} a_{n}(n+\delta) z^{n+\delta-1}\right] \\
& +\left[i \Omega\left(r_{+}-r_{-}\right) \frac{1+z}{1-z}-\Omega^{2} z \frac{\left(r_{+}-r_{-}\right)^{2}}{(1-z)^{2}}-l(l+1)+\frac{\left(r_{+}-z r_{-}\right)^{2}}{z\left(r_{+}-r_{-}\right)^{2}}\left(\omega\left(r_{-}+\frac{r_{+}-r_{-}}{1-z}\right)-q Q\right)^{2}-\mu^{2}\left(r_{-}+\frac{r_{+}-r_{-}}{1-z}\right)^{2}\right. \\
& \left.-i a m q Q\left(\frac{M}{r^{2}}-\frac{Q^{2}}{r^{3}}\right)+\operatorname{amq} Q \Omega \frac{\left(r_{+}-r_{-}\right)^{2} z}{\left(r_{+}-z r_{-}\right)^{2}}\right] \frac{\left(r_{+}-r_{-}\right)^{\epsilon}}{(1-z)^{\epsilon}} \sum_{n=0}^{\infty} a_{n} z^{n+\delta}=0 .
\end{aligned}
$$

After multiplying the whole relation with $(1-z)^{\epsilon}$, only a few terms with the fractions will remain. All these terms have either $1-z$ or its powers in the denominator. We combine them to get a simplified expression 


$$
\begin{aligned}
& \frac{2 \omega^{2} r_{-}\left(r_{+}-r_{-}\right)}{1-z} \sum_{n=0}^{\infty} a_{n} z^{n+\delta+1}+\left[2 \omega \frac{\left(r_{+}-r_{-}\right)\left(\omega r_{+}-q Q\right)}{1-z}+2 i \Omega \frac{\left(r_{+}-r_{-}\right) \epsilon z}{1-z}\right. \\
& \quad+i \Omega\left(r_{+}-r_{-}\right) \frac{1+z}{1-z}-\Omega^{2}\left(r_{+}-r_{-}\right)^{2} \frac{z}{(1-z)^{2}}+\omega^{2} \frac{\left(r_{+}-r_{-}\right)^{2} z}{(1-z)^{2}} \\
& \left.\quad-\mu^{2}\left(2 \frac{r_{-}\left(r_{+}-r_{-}\right)}{1-z}+\frac{\left(r_{+}-r_{-}\right)^{2}}{(1-z)^{2}}\right)\right] \sum_{n=0}^{\infty} a_{n} z^{n+\delta} \\
& =\sum_{n=0}^{\infty}\left[i \Omega\left(r_{+}-r_{-}\right)+2 \omega\left(\omega r_{+}-q Q\right)\left(r_{+}-r_{-}\right)-\mu^{2}\left(r_{+}+r_{-}\right)\left(r_{+}-r_{-}\right)\right] a_{n} z^{n+\delta} .
\end{aligned}
$$

We note that the relation $\Omega^{2}=\omega^{2}-\mu^{2}$ plays a crucial role in the above simplification and that the expression (4.7) for $\epsilon$ was utilized in the above calculation. Turning back to Eq. (A5), we make use of the simplification described above and obtain the following relation:

$$
\begin{aligned}
& \epsilon^{2} \sum_{n=0}^{\infty} a_{n} z^{n+\delta+1}+\epsilon \sum_{n=0}^{\infty} a_{n} z^{n+\delta}+\sum_{n=0}^{\infty} a_{n}(n+\delta) z^{n+\delta-1}+(2 \epsilon-2) \sum_{n=0}^{\infty} a_{n}(n+\delta) z^{n+\delta} \\
& +(1-2 \epsilon) \sum_{n=0}^{\infty} a_{n}(n+\delta) z^{n+\delta+1}+\sum_{n=0}^{\infty} a_{n}(n+\delta)(n+\delta-1) z^{n+\delta-1} \\
& -l(l+1) \sum_{n=0}^{\infty} a_{n} z^{n+\delta}-\mu^{2} r_{-}^{2} \sum_{n=0}^{\infty} a_{n} z^{n+\delta}-2 \sum_{n=0}^{\infty} a_{n}(n+\delta)(n+\delta-1) z^{n+\delta} \\
& +\sum_{n=0}^{\infty} a_{n}(n+\delta)(n+\delta-1) z^{n+\delta+1}+2 i \Omega\left(r_{+}-r_{-}\right) \sum_{n=0}^{\infty} a_{n}(n+\delta) z^{n+\delta} \\
& +\omega^{2} r_{-}^{2} \sum_{n=0}^{\infty} a_{n} z^{n+\delta+1}+\frac{2 \omega r_{-}^{2}}{r_{+}-r_{-}}\left(\omega r_{+}-q Q\right) \sum_{n=0}^{\infty} a_{n} z^{n+\delta}-\frac{2 \omega r_{-}^{2}}{r_{+}-r_{-}}\left(\omega r_{+}-q Q\right) \sum_{n=0}^{\infty} a_{n} z^{n+\delta+1} \\
& +\frac{r_{-}^{2}}{\left(r_{+}-r_{-}\right)^{2}}\left(\omega r_{+}-q Q\right)^{2} \sum_{n=0}^{\infty} a_{n} z^{n+\delta-1}-2 \frac{r_{-}^{2}}{\left(r_{+}-r_{-}\right)^{2}}\left(\omega r_{+}-q Q\right)^{2} \sum_{n=0}^{\infty} a_{n} z^{n+\delta} \\
& +\frac{r_{-}^{2}}{\left(r_{+}-r_{-}\right)^{2}}\left(\omega r_{+}-q Q\right)^{2} \sum_{n=0}^{\infty} a_{n} z^{n+\delta+1}+4 \omega r_{-}\left(\omega r_{+}-q Q\right) \sum_{n=0}^{\infty} a_{n} z^{n+\delta} \\
& +\frac{2 r_{-}}{r_{+}-r_{-}}\left(\omega r_{+}-q Q\right)^{2} \sum_{n=0}^{\infty} a_{n} z^{n+\delta-1}-\frac{2 r_{-}}{r_{+}-r_{-}}\left(\omega r_{+}-q Q\right)^{2} \sum_{n=0}^{\infty} a_{n} z^{n+\delta} \\
& +\left(\omega r_{+}-q Q\right)^{2} \sum_{n=0}^{\infty} a_{n} z^{n+\delta-1}-i a m q Q\left(r_{+}-r_{-}\right) \frac{(1-z)^{2}}{\left(r_{+}-z r_{-}\right)^{2}} \sum_{n=0}^{\infty} a_{n}(n+\delta) z^{n+\delta} \\
& +\sum_{n=0}^{\infty} a_{n} z^{n+\delta}\left[i \Omega\left(r_{+}-r_{-}\right)+2 \omega\left(\omega r_{+}-q Q\right)\left(r_{+}-r_{-}\right)-\mu^{2}\left(r_{+}+r_{-}\right)\left(r_{+}-r_{-}\right)\right] \\
& -\operatorname{iamq} Q \frac{\left(r_{+}-r_{-}\right) \epsilon(1-z)}{\left(r_{+}-z r_{-}\right)^{2}} \sum_{n=0}^{\infty} a_{n} z^{n+\delta+1}-\operatorname{iam} q Q \frac{(1-z)^{2}}{\left(r_{+}-z r_{-}\right)^{2}}\left(M-Q^{2} \frac{1-z}{r_{+}-z r_{-}}\right) \sum_{n=0}^{\infty} a_{n} z^{n+\delta} \\
& +\operatorname{amq} Q \Omega \frac{\left(r_{+}-r_{-}\right)^{2}}{\left(r_{+}-z r_{-}\right)^{2}} \sum_{n=0}^{\infty} a_{n} z^{n+\delta+1}=0,
\end{aligned}
$$

where $2 M=r_{+}+r_{-}$and $Q^{2}=r_{+} r_{-}$. Terms that do not involve the deformation parameter $a$ may combine [32,33] into an expansion leading to a three-term recurrence relation. In this way (A7) is brought to the form 


$$
\begin{aligned}
& \left(r_{+}-z r_{-}\right)^{3} \sum_{n=1}^{\infty}\left[\alpha_{n} a_{n+1}+\beta_{n} a_{n}+\gamma_{n} a_{n-1}\right] z^{n+\delta}+\left(r_{+}-z r_{-}\right)^{3}\left(\alpha_{0} a_{1}+\beta_{0} a_{0}\right) z^{\delta} \\
& \quad+\left(r_{+}-z r_{-}\right)^{3}\left[\delta^{2}+\left(\omega r_{+}-q Q\right)^{2} \frac{r_{+}^{2}}{\left(r_{+}-r_{-}\right)^{2}}\right] a_{0} z^{\delta-1} \\
& \quad-i a m q Q\left(r_{+}-r_{-}\right) \epsilon\left(r_{+}-z r_{-}\right)(1-z) \sum_{n=0}^{\infty} a_{n} z^{n+\delta+1} \\
& \quad-i a m q Q\left(r_{+}-r_{-}\right)\left(r_{+}-z r_{-}\right)(1-z)^{2} \sum_{n=0}^{\infty} a_{n}(n+\delta) z^{n+\delta} \\
& \quad-i a m q Q\left(r_{+}-z r_{-}\right)(1-z)^{2} \sum_{n=0}^{\infty} a_{n} z^{n+\delta} \\
& \quad+i a m q Q r_{+} r_{-}(1-z)^{3} \sum_{n=0}^{\infty} a_{n} z^{n+\delta}+a m q Q \Omega\left(r_{+}-r_{-}\right)^{2}\left(r_{+}-z r_{-}\right) \sum_{n=0}^{\infty} a_{n} z^{n+\delta+1}=0,
\end{aligned}
$$

with $\alpha_{n}, \beta_{n}, \gamma_{n}$ being written in (4.10). Since the general solution (4.6) has to be nonsingular at the horizon $z=0$, and $z^{\delta-1}$ is a term of the lowest order in $z$, its coefficient in (A8) has to vanish. This confirms the condition on $\delta$ that was obtained previously by matching with the QNM boundary conditions; see (4.7).

Next, we group together terms with the same power of $z$ and shift the summation indices where needed to bring all terms into a form having the same generic power $z^{n+\delta}$. Hence, we note that all terms determined by $n \geq 4$ give rise to the general six-term recurrence relation which characterizes the problem studied in this paper. Along with that, we need to sort out all the remaining terms that do not fall into the above category. These terms give rise to the indicial recurrence relations. They are obtained by analyzing the coefficients that, respectively, stand next to the powers $z^{\delta}, z^{\delta+1}, z^{\delta+2}$, and $z^{\delta+3}$. They may be considered as the boundary conditions imposed on the main, i.e., the generic recurrence relation. The result is the following relation:

$$
\begin{aligned}
\sum_{n=4}^{\infty} & {\left[A_{n} a_{n+1}+B_{n} a_{n}+C_{n} a_{n-1}+D_{n} a_{n-2}+E_{n} a_{n-3}+F_{n} a_{n-4}\right] z^{n+\delta} } \\
& +\left[\left(3 r_{+} r_{-}^{2} \gamma_{1}+i a m q Q\left(r_{+}-r_{-}\right) r_{-} \delta-r_{-}^{3} \beta_{0}-i a m q Q\left(r_{+}-r_{-}\right) r_{-} \epsilon+i a m q Q M r_{-}\right.\right. \\
& \left.-i a m q Q r_{+} r_{-}\right) a_{0}+\left(3 r_{+} r_{-}^{2} \beta_{1}+i a m q Q\left(r_{+}^{2}-r_{-}^{2}\right) \epsilon-i a m q Q\left(r_{+}-r_{-}\right)\left(r_{+}+2 r_{-}\right)(\delta+1)\right. \\
& \left.-i a m q Q M\left(r_{+}+2 r_{-}\right)+3 i a m q Q r_{+} r_{-}-a m q Q \Omega\left(r_{+}-r_{-}\right)^{2} r_{-}-3 r_{+}^{2} r_{-} \gamma_{2}-r_{-}^{3} \alpha_{0}\right) a_{1} \\
& +\left(r_{+}^{3} \gamma_{3}+i a m q Q\left(r_{+}-r_{-}\right)\left(2 r_{+}-r_{-}\right)(\delta+2)-3 r_{+}^{2} r_{-} \beta_{2}-i a m q Q\left(r_{+}-r_{-}\right) r_{+} \epsilon\right. \\
& \left.+i a m q Q M\left(2 r_{+}+r_{-}\right)-3 i a m q Q r_{+} r_{-}+a m q Q \Omega\left(r_{+}-r_{-}\right)^{2} r_{+}+3 r_{+} r_{-}^{2} \alpha_{1}\right) a_{2} \\
& \left.+\left(r_{+}^{3} \beta_{3}-i a m q Q\left(r_{+}-r_{-}\right) r_{+}(\delta+3)-i a m q Q M r_{+}+i a m q Q r_{+} r_{-}-3 r_{+}^{2} r_{-} \alpha_{2}\right) a_{3}+r_{+}^{3} \alpha_{3} a_{4}\right] z^{\delta+3} \\
& +\left[\left(3 r_{+} r_{-}^{2} \beta_{0}+i a m q Q\left(r_{+}-r_{-}\right)\left(r_{+}+r_{-}\right) \epsilon-i a m q Q\left(r_{+}-r_{-}\right)\left(r_{+}+2 r_{-}\right) \delta\right.\right. \\
& \left.-i a m q Q M\left(r_{+}+2 r_{-}\right)+3 i a m q Q r_{+} r_{-}-a m q Q \Omega\left(r_{+}-r_{-}\right)^{2} r_{-}-3 r_{+}^{2} r_{-} \gamma_{1}\right) a_{0} \\
& +\left(r_{+}^{3} \gamma_{2}+i a m q Q\left(r_{+}-r_{-}\right)\left(2 r_{+}+r_{-}\right)(\delta+1)-3 r_{+}^{2} r_{-} \beta_{1}-i a m q Q\left(r_{+}-r_{-}\right) r_{+} \epsilon\right. \\
& \left.+i a m q Q M\left(2 r_{+}+r_{-}\right)-3 i a m q Q r_{+} r_{-}+a m q Q \Omega\left(r_{+}-r_{-}\right)^{2} r_{+}+3 r_{+} r_{-}^{2} \alpha_{0}\right) a_{1} \\
& \left.+\left(r_{+}^{3} \beta_{2}-i a m q Q\left(r_{+}-r_{-}\right) r_{+}(\delta+2)-i a m q Q M r_{+}-3 r_{+}^{2} r_{-} \alpha_{1}+i a m q Q r_{+} r_{-}\right) a_{2}+r_{+}^{3} \alpha_{2} a_{3}\right] z^{\delta+2} \\
& +\left[\left(r_{+}^{3} \gamma_{1}+a m q Q \Omega\left(r_{+}-r_{-}\right)^{2} r_{+}-3 r_{+}^{2} r_{-} \beta_{0}-i a m q Q\left(r_{+}-r_{-}\right) r_{+} \epsilon\right.\right. \\
& \left.+i a m q Q\left(r_{+}-r_{-}\right)\left(2 r_{+}+r_{-}\right) \delta+i a m q Q M\left(2 r_{+}+r_{-}\right)-3 i a m q Q r_{+} r_{-}\right) a_{0} \\
& \left.+\left(r_{+}^{3} \beta_{1}-i a m q Q\left(r_{+}-r_{-}\right) r_{+}(\delta+1)-i a m q Q M r_{+}+i a m q Q r_{+} r_{-}-3 r_{+}^{2} r_{-} \alpha_{0}\right) a_{1}+r_{+}^{3} \alpha_{1} a_{2}\right] z^{\delta+1} \\
& +\left[\left(r_{+}^{3} \beta_{0}+i a m q Q r_{+} r_{-}-i a m q Q\left(r_{+}-r_{-}\right) r_{+} \delta-i a m q Q M r_{+}\right) a_{0}+r_{+}^{3} \alpha_{0} a_{1}\right] z^{\delta}=0 .
\end{aligned}
$$


The coefficients $A_{n}, B_{n}, C_{n}, D_{n}, E_{n}, F_{n}$ that appear here are given in (4.9). Moreover, it is easy to see that the expressions multiplying $a_{0}, a_{1}, a_{2}, a_{3}, a_{4}$ that appear in front of the lowest order powers $z^{\delta}, z^{\delta+1}, z^{\delta+2}$, and $z^{\delta+3}$ may be recognized as the particular cases of the coefficients $A_{n}, B_{n}, C_{n}, D_{n}, E_{n}, F_{n}$. Indeed, these expressions may be written in terms of $A_{i}, B_{i}, C_{i}, D_{i}, E_{i}, i=0,1,2,3$. Thus, the sequence of recurrence relations following from (A9) can be written as

$$
\begin{aligned}
& A_{n} a_{n+1}+B_{n} a_{n}+C_{n} a_{n-1}+D_{n} a_{n-2}+E_{n} a_{n-3}+F_{n} a_{n-4}=0, \\
& A_{3} a_{4}+B_{3} a_{3}+C_{3} a_{2}+D_{3} a_{1}+E_{3} a_{0}=0, \\
& A_{2} a_{3}+B_{2} a_{2}+C_{2} a_{1}+D_{2} a_{0}=0, \\
& A_{1} a_{2}+B_{1} a_{1}+C_{1} a_{0}=0, \\
& A_{0} a_{1}+B_{0} a_{0}=0 .
\end{aligned}
$$

\section{APPENDIX B: GAUSS ELIMINATION PROCEDURE}

In the following we explain the required steps in a Gauss elimination procedure applied to our particular model. We need to reduce the six-term recurrence relation (4.8) to a three-term recurrence relation.

To begin with, we define the coefficients of the zeroth level $A_{n}^{(0)}, B_{n}^{(0)}, C_{n}^{(0)}, D_{n}^{(0)}, E_{n}^{(0)}, F_{n}^{(0)}$ to be the coefficients of the initial six-term recurrence relation (4.8),

$$
A_{n}^{(0)} \equiv A_{n}, \quad B_{n}^{(0)} \equiv B_{n}, \quad C_{n}^{(0)} \equiv C_{n}, \quad D_{n}^{(0)} \equiv D_{n}, \quad E_{n}^{(0)} \equiv E_{n}, \quad F_{n}^{(0)} \equiv F_{n},
$$

with $A_{n}, B_{n}, C_{n}, D_{n}, E_{n}, F_{n}$ defined in (4.9). Similarly, we introduce the coefficients of the $j$ th level $A_{n}^{(j)}, B_{n}^{(j)}, C_{n}^{(j)}, D_{n}^{(j)}$, $E_{n}^{(j)}, j=1,2,3$ as the coefficients that appear in the $(6-j)$-term recurrence relation, obtained after the $j$ th Gauss elimination.

Applying the first Gauss elimination to (4.8) we find the five-term recurrence relation

$$
\begin{aligned}
A_{n}^{(1)} a_{n+1}+B_{n}^{(1)} a_{n}+C_{n}^{(1)} a_{n-1}+D_{n}^{(1)} a_{n-2}+E_{n}^{(1)} a_{n-3} & =0, \\
A_{2}^{(1)} a_{3}+B_{2}^{(1)} a_{2}+C_{2}^{(1)} a_{1}+D_{2}^{(1)} a_{0} & =0, \\
A_{1}^{(1)} a_{2}+B_{1}^{(1)} a_{1}+C_{1}^{(1)} a_{0} & =0, \\
A_{0}^{(1)} a_{1}+B_{0}^{(1)} a_{0} & =0 .
\end{aligned}
$$

The coefficients of the first level are determined as

$$
\begin{array}{ll}
A_{n}^{(1)}=A_{n}^{(0)}, \quad n \geq 4, & \\
B_{n}^{(1)}=B_{n}^{(0)}-\frac{F_{n}^{(0)}}{E_{n-1}^{(1)}} A_{n-1}^{(1)}, \quad C_{n}^{(1)}=C_{n}^{(0)}-\frac{F_{n}^{(0)}}{E_{n-1}^{(1)}} B_{n-1}^{(1)}, \\
D_{n}^{(1)}=D_{n}^{(0)}-\frac{F_{n}^{(0)}}{E_{n-1}^{(1)}} C_{n-1}^{(1)}, & E_{n}^{(1)}=E_{n}^{(0)}-\frac{F_{n}^{(0)}}{E_{n-1}^{(1)}} D_{n-1}^{(1)},
\end{array}
$$

and for $n=3,2,1,0$,

$$
\begin{aligned}
& A_{3}^{(1)}=A_{3}^{(0)}, \quad B_{3}^{(1)}=B_{3}^{(0)}, \quad C_{3}^{(1)}=C_{3}^{(0)}, \quad D_{3}^{(1)}=D_{3}^{(0)}, \quad E_{3}^{(1)}=E_{3}^{(0)}, \\
& A_{2}^{(1)}=A_{2}^{(0)}, \quad B_{2}^{(1)}=B_{2}^{(0)}, \quad C_{2}^{(1)}=C_{2}^{(0)}, \quad D_{2}^{(1)}=D_{2}^{(0)}, \\
& A_{1}^{(1)}=A_{1}^{(0)}, \quad B_{1}^{(1)}=B_{1}^{(0)}, \quad C_{1}^{(1)}=C_{1}^{(0)}, \\
& A_{0}^{(1)}=A_{0}^{(0)}, \quad B_{0}^{(1)}=B_{0}^{(0)} \text {. }
\end{aligned}
$$

The application of the second Gauss elimination to the recurrence relation (B2) yields the following four-term recurrence equation: 


$$
\begin{aligned}
A_{n}^{(2)} a_{n+1}+B_{n}^{(2)} a_{n}+C_{n}^{(2)} a_{n-1}+D_{n}^{(2)} a_{n-2} & =0, \\
A_{1}^{(2)} a_{2}+B_{1}^{(2)} a_{1}+C_{1}^{(2)} a_{0} & =0, \\
A_{0}^{(2)} a_{1}+B_{0}^{(2)} a_{0} & =0 .
\end{aligned}
$$

The coefficients of the second level are determined as

$$
\begin{aligned}
& A_{n}^{(2)}=A_{n}^{(1)}=A_{n}^{(0)}, \quad B_{n}^{(2)}=B_{n}^{(1)}-\frac{E_{n}^{(1)}}{D_{n-1}^{(2)}} A_{n-1}^{(2)}, \quad n \geq 3, \\
& C_{n}^{(2)}=C_{n}^{(1)}-\frac{E_{n}^{(1)}}{D_{n-1}^{(2)}} B_{n-1}^{(2)}, \quad D_{n}^{(2)}=D_{n}^{(1)}-\frac{E_{n}^{(1)}}{D_{n-1}^{(2)}} C_{n-1}^{(2)},
\end{aligned}
$$

and for $n=0,1,2$,

$$
\begin{array}{llll}
A_{2}^{(2)}=A_{2}^{(1)}=A_{2}^{(0)}, & B_{2}^{(2)}=B_{2}^{(1)}=B_{2}^{(0)}, & C_{2}^{(2)}=C_{2}^{(1)}=C_{2}^{(0)}, & D_{2}^{(2)}=D_{2}^{(1)}=D_{2}^{(0)}, \\
A_{1}^{(2)}=A_{1}^{(1)}=A_{1}^{(0)}, & B_{1}^{(2)}=B_{1}^{(1)}=B_{1}^{(0)}, & C_{1}^{(2)}=C_{1}^{(1)}=C_{1}^{(0)}, \\
A_{0}^{(2)}=A_{0}^{(1)}=A_{0}^{(0)}, & B_{0}^{(2)}=B_{0}^{(1)}=B_{0}^{(0)} . &
\end{array}
$$

Third, the last Gauss elimination applied to (B5) leads to the recurrence relation

$$
\begin{aligned}
A_{n}^{(3)} a_{n+1}+B_{n}^{(3)} a_{n}+C_{n}^{(3)} a_{n-1} & =0, \\
A_{0}^{(3)} a_{1}+B_{0}^{(3)} a_{0} & =0 .
\end{aligned}
$$

The coefficients of the third level, $A_{n}^{(3)}, B_{n}^{(3)}, C_{n}^{(3)}$, that we have been searching for, are given by

$$
\begin{aligned}
& A_{n}^{(3)}=A_{n}^{(2)}=A_{n}^{(0)}, \quad n \geq 2, \\
& B_{n}^{(3)}=B_{n}^{(2)}-\frac{D_{n}^{(2)}}{C_{n-1}^{(3)}} A_{n-1}^{(3)}, \quad C_{n}^{(3)}=C_{n}^{(2)}-\frac{D_{n}^{(2)}}{C_{n-1}^{(3)}} B_{n-1}^{(3)},
\end{aligned}
$$

and for $n=0,1$,

$$
\begin{array}{lll}
A_{1}^{(3)}=A_{1}^{(2)}=A_{1}^{(0)}, & B_{1}^{(3)}=B_{1}^{(2)}=B_{1}^{(0)}, & C_{1}^{(3)}=C_{1}^{(2)}=C_{1}^{(0)}, \\
A_{0}^{(3)}=A_{0}^{(2)}=A_{0}^{(0)}, & B_{0}^{(3)}=B_{0}^{(2)}=B_{0}^{(0)} . &
\end{array}
$$

[1] T. Regge and J. A. Wheeler, Stability of a Schwarzschild singularity, Phys. Rev. 108, 1063 (1957).

[2] C. V. Vishveshwara, Scattering of gravitational radiation by a Schwarzschild black-hole, Nature (London) 227, 936 (1970).

[3] B. P. Abbott et al., Observation of Gravitational Waves from a Binary Black Hole Merger, Phys. Rev. Lett. 116, 061102 (2016).
[4] W. H. Press, Long wave trains of gravitational waves from a vibrating black hole, Astrophys. J. 170, L105 (1971).

[5] H. P. Nollert, Quasinormal modes: The characteristic "sound" of black holes and neutron stars, Classical Quantum Gravity 16, R159 (1999).

[6] K. D. Kokkotas and B. G. Schmidt, Quasinormal modes of stars and black holes, Living Rev. Relativity 2, 2 (1999). 
[7] E. Berti, V. Cardoso, and A. O. Starinets, Quasinormal modes of black holes and black branes, Classical Quantum Gravity 26, 163001 (2009).

[8] R. A. Konoplya and A. Zhidenko, Quasinormal modes of black holes: From astrophysics to string theory, Rev. Mod. Phys. 83, 793 (2011).

[9] V. Cardoso and J. P. S. Lemos, Scalar, electromagnetic and Weyl perturbations of BTZ black holes: Quasinormal modes, Phys. Rev. D 63, 124015 (2001).

[10] V. Cardoso and J.P. S. Lemos, Quasinormal modes of Schwarzschild anti-de Sitter black holes: Electromagnetic and gravitational perturbations, Phys. Rev. D 64, 084017 (2001).

[11] B. F. Schutz and C. M. Will, Black hole normal modes: A semianalytic approach, Astrophys. J. 291, L33 (1985).

[12] S. Iyer and C. M. Will, Black hole normal modes: A WKB approach. 1. Foundations and application of a higher order WKB analysis of potential barrier scattering, Phys. Rev. D 35, 3621 (1987).

[13] V. Ferrari and B. Mashhoon, New approach to the quasinormal modes of a black hole, Phys. Rev. D 30, 295 (1984).

[14] S. Chandrasekhar and S. L. Detweiler, The quasi-normal modes of the Schwarzschild black hole, Proc. R. Soc. A 344, 441 (1975).

[15] C. Gundlach, R. H. Price, and J. Pullin, Late time behavior of stellar collapse and explosions: 2. Nonlinear evolution Phys. Rev. D 49, 883 (1994).

[16] K. S. Gupta, E. Harikumar, T. Jurić, S. Meljanac, and A. Samsarov, Noncommutative scalar quasinormal modes and quantization of entropy of a BTZ black hole, J. High Energy Phys. 09 (2015) 025.

[17] K. S. Gupta, T. Jurić, and A. Samsarov, Noncommutative duality and fermionic quasinormal modes of the BTZ black hole, J. High Energy Phys. 06 (2017) 107.

[18] M. D. Ćirić, N. Konjik, and A. Samsarov, Noncommutative scalar quasinormal modes of the Reissner-Nordström black hole, Classical Quantum Gravity 35, 175005 (2018).

[19] A. Connes, Non-Commutative Geometry (Academic Press, New York, 1994); G. Landi, An Introduction to Noncommutative Spaces and their Geometry, Lecture Notes Physics Monographs Vol. 51 (Springer, New York, 1997); J. Madore, An Introduction to Noncommutative Differential Geometry and its Physical Applications, 2nd ed. (Cambridge University Press, Cambridge, England, 1999).

[20] P. Aschieri, M. Dimitrijević, P. Kulish, F. Lizzi, and J. Wess, Noncommutative Spacetimes: Symmetries in Noncommutative Geometry and Field Theory, Lecture Notes in Physics Vol. 774 (Springer, New York, 2009).

[21] M. Dimitrijević Ćirić, N. Konjik, M. A. Kurkov, F. Lizzi, and P. Vitale, Noncommutative field theory from angular twist, Phys. Rev. D 98, 085011 (2018).

[22] M. Dimitrijević Ćirić, B. Nikolić, and V. Radovanović, Noncommutative gravity and the relevance of the thetaconstant deformation, Europhys. Lett. 118, 21002 (2017).

[23] P. Aschieri and L. Castellani, Noncommutative $D=4$ gravity coupled to fermions J. High Energy Phys. 06 (2009) 086.
[24] P. Aschieri and L. Castellani, Noncommutative gravity coupled to fermions: Second order expansion via Seiberg-Witten map, J. High Energy Phys. 07 (2012) 184.

[25] S. Hod, Relaxation dynamics of charged gravitational collapse, Phys. Lett. A 374, 2901 (2010).

[26] S. Hod, Quasinormal resonances of a charged scalar field in a charged Reissner-Nordström black hole spacetime: A WKB analysis, Phys. Lett. B 710, 349 (2012).

[27] P. Kraus and F. Wilczek, Some applications of a simple stationary line element for the Schwarzschild geometry, Mod. Phys. Lett. A 09, 3713 (1994); M. K. Parikh and F. Wilczek, Hawking Radiation as Tunneling, Phys. Rev. Lett. 85, 5042 (2000).

[28] R. A. Konoplya and A. Zhidenko, Massive charged scalar field in the Kerr-Newman background I: Quasinormal modes, late-time tails and stability, Phys. Rev. D 88, 024054 (2013).

[29] R. A. Konoplya, Quasinormal behavior of the d-dimensional Schwarzschild black hole and higher order WKB approach, Phys. Rev. D 68, 024018 (2003).

[30] E. W. Leaver, An analytic representation for the quasi normal modes of Kerr black holes, Proc. R. Soc. A 402, 285 (1985).

[31] H. P. Nollert, Quasinormal modes of Schwarzschild black holes: The determination of quasinormal frequencies with very large imaginary parts, Phys. Rev. D 47, 5253 (1993).

[32] M. Richartz and D. Giugno, Quasinormal modes of charged fields around a Reissner-Nordström black hole, Phys. Rev. D 90, 124011 (2014).

[33] A. Chowdhury and N. Banerjee, Quasinormal modes of a charged spherical black hole with scalar hair for scalar and Dirac perturbations, Eur. Phys. J. C 78, 594 (2018).

[34] W. Gautschi, Computational aspects of three-term recurrence relations, SIAM Rev. 9, 24 (1967).

[35] H. Onozawa, T. Mishima, T. Okamura, and H. Ishihara, Quasinormal modes of maximally charged black holes, Phys. Rev. D 53, 7033 (1996).

[36] S. Ray, A. L. Espindola, M. Malheiro, J. P. S. Lemos, and V. T. Zanchin, Electrically charged compact stars and formation of charged black holes, Phys. Rev. D 68, 084004 (2003).

[37] M. Dimitrijević Ćirić, B. Nikolić, and V. Radovanović, NC $S O(2,3)$ ^ gravity: Noncommutativity as a source of curvature and torsion, Phys. Rev. D 96, 064029 (2017).

[38] P. Nicolini, Noncommutative black holes, the final appeal to quantum gravity: A review, Int. J. Mod. Phys. A 24, 1229 (2009); L. Modesto and P. Nicolini, Charged rotating noncommutative black holes, Phys. Rev. D 82, 104035 (2010).

[39] A. Smailagic and E. Spallucci, Feynman path integral on the noncommutative plane, J. Phys. A 36, L467 (2003); UV divergence free QFT on noncommutative plane, J. Phys. A 36, L517 (2003).

[40] A. M. Perelomov, Coherent states for arbitrary Lie groups, Commun. Math. Phys. 26, 222 (1972). 